hep-th/0409058

UT-KOMABA/04-10

September 2004

\title{
Impurity Non-Preserving 3-Point Correlators of BMN Operators from PP-Wave Holography I : Bosonic Excitations
}

\author{
Suguru DoBAshi * and Tamiaki YoneYA ${ }^{\dagger}$ \\ Institute of Physics, University of Tokyo \\ Komaba, Meguro-ku, Tokyo 153-8902
}

\begin{abstract}
As a continuation of our previous works studying the holographic principle in the planewave limit, we discuss the 3-point correlation functions of BMN operators with bosonic excitations when impurities are not conserved. We show that our proposal for a holographic mapping between the conformal OPE coefficients of super Yang-Mills theory and the 3-point vertex of the holographic string field theory is valid to the leading order in the large $\mu$ limit. Our results provide for the first time a direct holographic relation for the 3-point correlators of BMN operators including impurity non-preserving processes.
\end{abstract}

*e-mail address: doba@hep1.c.u-tokyo.ac.jp

${ }^{\dagger}$ e-mail address: tam@hep1.c.u-tokyo.ac.jp 


\section{Introduction}

What is the correct interpretation of holographic principle in the PP-wave limit of the $\mathrm{AdS}_{5} / \mathrm{SYM}_{4}$ correspondence has been one among several open problems since the first original discussion [1] of the BMN limit. In the previous paper [2], we proposed a simple direct relation between the 3-point OPE coefficients of conformal BMN operators and the bulk 3-point interaction vertex of string field theory, by developing the basic ideas presented first in ref. [3]. In [2], we constructed the 'holographic' string field theory which meets requirements for the validity of the GKP-Witten relation and confirmed explicitly that it indeed reproduces the 3-point OPE coefficients of $\mathcal{N}=4$ super Yang-Mills theory in the leading large- $\mu$ expansion for stringy BMN operators in the impurity-preserving sector. Furthermore, it was also clarified that two entirely different proposals for relating 3-point vertices of (different versions of) string field theories to corresponding quantities on the gauge-theory side in the impuritiy-preserving sector are actually compatible to each other through our holographic map.

It is important to recall, as we have stressed there, that our holographic relation in principle should be valid for much more general impurity non-preserving processes too, since by construction the holographic relation is satisfied for the so-called 'non-extremal' 3-point functions of chiral primary operators in the BMN limit. The latter functions are regarded to be the supergravity sector of general impurity non-preserving 3-point functions. The goal of the present paper is to confirm that our stringy holographic relation is indeed satisfied for impurity non-preserving cases in the leading large- $\mu$ expansion, which is at the same level as in the existing treatments of the 3-point functions of impuritypreserving sector on both sides.

The significance of this result seems evident, since the usual discussions in most of other works being focused almost exclusively on the dilatation operator are practically restricted to impurity-preserving sector and therefore are difficult to extend them to impurity non-preserving processes. ${ }^{\ddagger}$ In contrast to this, the string-field Hamiltonian in our approach cannot exactly be interpreted as the dilatation operator, since it generates an infinitesimal translation along a geodesic connecting from boundary to boundary in the

\footnotetext{
${ }^{\ddagger}$ On the gauge-theory side, the matrix elements of a dilatation operator in the sense of renormalized perturbation theory would vanish unless the states have degenerate conformal dimensions in the lowest order. This is borne out in computations in refs. 99 [11, 10].
} 
Euclidean AdS spacetime. Except for regions approaching asymptotically to the conformal boundary, such a translation cannot be identified with dilatation. In fact, this discrepancy between bulk Hamiltonian and dilatation operator on the boundary is, in our opinion, even more so in the usual derivation of the Penrose limit, leading to a geodesic which is disconnected from the boundary: There would be no asymptotic region where the Hamiltonian is related to the dilatation, corresponding to the fact that the translation with respect to the global time coordinate of the AdS spacetime does not coincide with dilatation on the boundary at least within the usual geometric interpretation of the AdS spacetimes. This puzzle was our main motivation for undertaking the present series of works [3, 4, 5], to which we would like to invite the reader for further discussions of this basic issue. From our viewpoint, the usual Penrose limit is obtained formally by a Wick rotation of the affine time parameter along the geodesics connecting boundary to boundary. Note that the relative topology of trajectory with respect to the conformal boundary is completely changed by this Wick rotation. This however explains why the dilatation comes about even in Minkowski formulation. In any case, given the apparent correspondence for conformal dimensions $\left\{\Delta_{r}\right\}$ on one hand, it would be very strange if there could be no clear way of mapping the OPE coefficients which, together with conformal dimensions, constitute the crucial data of CFT.

We note that, even though the matrix elements of 3-point interaction vertices of string field theory in the impurity non-preserving sector often have additional powers in $1 / \mu, \S$ the OPE coefficents are not necessarily of lower order in the large- $\mu$ expansion for large but fixed $R$-charge angular momentum $J$. It is also well known that a class of impurity non-preserving processes cannot simply be ignored in summing up intermediate states in higher-loop calculations, at least within the logic of string field theory as presently understood. Once the summation over intermediate states are involved, the limiting procedures required in the studies of amplitudes for BMN states should be a very subtle problem, since results would depend on the orders of various infinite sums and the limiting procedures. We hope that our concrete results for impurity non-preserving 3-point correlators provide a useful first step for further exploration of the AdS holography in the plane-wave limit beyond the approximation of keeping only the impurity preserving sector.

\footnotetext{
$\S$ This is due to an additional power of $1 / \mu$ in the asymptotic forms of Neumann functions other than $\tilde{N}_{m n}^{21}$ and $\tilde{N}_{m n}^{31}$. See the Appendix.
} 
In the next section, we start from summarizing briefly the holographic relation and the holographic string field theory proposed in the previous work. In section 3, we treat a general case of impurity non-preserving 3-point functions where only non-singlet (more precisely, traceless) scalar (and bosonic) impurities corresponding to the fluctuations along 4-directions among the $S^{5}$ of $\mathrm{AdS}_{5} \times S^{5}$ are involved. In section 4, we extend the results to include non-singlet vector excitations. In section 5, we discuss a few typical cases where singlet impurities are involved. The concluding section 6 contains some remarks on relevant issues, especially, the uniqueness of our holographic relation and the question of higher-order effects. Some useful formulas which are relevant for discussions in the main text are summarized in the Appendix.

\section{The holographic relation and string field theory}

The key concept behind our holographic relation is that the correspondence between conformal boundary and bulk spacetime in the plane-wave limit of the AdS/CFT correspondence should be based on a tunneling geodesics traversing inside the AdS spacetime with the Euclidean signature which start from the conformal boundary and end again at conformal boundary, instead of the usual procedure of taking the Penrose limit in the AdS space-time with Minkowski signature. Such amplitudes can be regarded as an Euclidean analog of the usual S-matrix. This picture, which has been previously proposed by us for the purpose of resolving some puzzles associated with holography in the PP-wave limit in [3], automatically emerges by studying the large- $J$ limit of the GKP-Witten relation for single-trace chiral primary operators. Since the 3-point functions of chiral primary operators are protected against the perturbative corrections on the gauge-theory side, we can derive the effective action describing the interaction of the BMN operators along the tunneling geodesics in the supergravity sector. The full string field theory, the 'holographic' string field theory, should reduce to this effective action in the supergravity sector.

Under this requirement, we are led to a unique 3-point vertex which should obey the holographic relation based on the above tunneling picture. We stress that in the GKP-Witten conjecture the bulk partition function as a functional of boundary fields is fixed uniquely with a given basis of linearized supergravity fields, independently of field redefinitions. For 3-point correlators, the effect of such a field redefinition would be proportional to the equation of motion, and integration over the interaction point in 
the bulk would give vanishing result. In this sense, even though 'energies' being now the conformal dimensions are not conserved, the Euclidean S-matrix is very similar [3] to the ordinary S-matrix of flat Minkowski spacetime. We will give a remark on the issue of uniqueness of our prescription in the final section of the present paper. A subtlety in the case of the extremal case where the conformal dimensions are strictly conserved will also be clarified.

The holographic relation is summarized as

$$
\begin{gathered}
C_{123}=\frac{\tilde{\lambda}_{123}}{\mu\left(\Delta_{2}+\Delta_{3}-\Delta_{1}\right)}, \\
\tilde{\lambda}_{123}=\left(f \frac{J_{2} J_{3}}{J_{1}}\right)^{-\left(\Delta_{2}+\Delta_{3}-\Delta_{1}\right) / 2} \Gamma\left(\frac{\Delta_{2}+\Delta_{3}-\Delta_{1}}{2}+1\right) \lambda_{123}
\end{gathered}
$$

with

$$
\lambda_{123}={ }_{(1)}\left\langle1 | _ { ( 2 ) } \left\langle\left. 2\right|_{(3)}\left\langle 3\left|\frac{\sqrt{J_{1} J_{2} J_{3}}}{N}\right| H_{3}\right\rangle_{h},\right.\right.
$$

The conformal dimensions $\Delta_{r}$ are those in the planar limit. The CFT coefficients $C_{123}$ is defined under the standard normalization of two point functions as

$$
\left\langle\bar{O}_{1}\left(\vec{x}_{1}\right) O_{2}\left(\vec{x}_{2}\right)\right\rangle=\frac{\delta_{12}}{\left|\vec{x}_{12}\right|^{2 \Delta_{1}}}
$$

by

$$
\left\langle\bar{O}_{1}\left(\vec{x}_{1}\right) O_{2}\left(\vec{x}_{2}\right) O_{3}\left(\vec{x}_{3}\right)\right\rangle=\frac{C_{123}}{\left|\vec{x}_{12}\right|^{2 \alpha_{3}}\left|\vec{x}_{23}\right|^{2 \alpha_{1}}\left|\vec{x}_{31}\right|^{2 \alpha_{2}}}
$$

with $\left(\vec{x}_{12}=\vec{x}_{1}-\vec{x}_{2}\right.$, etc $)$

$$
\alpha_{1}=\frac{\Delta_{2}+\Delta_{3}-\Delta_{1}}{2}, \quad \text { etc. }
$$

The symbol $\left|H_{3}\right\rangle_{h}$ is the 3-point interaction vertex of the holographic string field theory

$$
S_{3}=\frac{1}{2} \int d \tau_{(1)}\left\langle\overline { \psi } | _ { ( 2 ) } \left\langle\left.\psi\right|_{(3)}\left\langle\psi\left|\frac{\sqrt{J_{1} J_{2} J_{3}}}{N}\right| H_{3}\right\rangle_{h}+\right.\right.\text { h.c. . }
$$

Here the integration over the $R$-charge angular momenta $J_{r}$ associated with the string fields ${ }_{(r)}\langle\psi|(r=2,3),{ }_{(1)}\langle\bar{\psi}|$ should be implicitly understood, under the conservation condition $J_{1}=J_{2}+J_{3}$. The quantity $f$ in (2.2) depending on $\alpha^{\prime}$ is responsible for the nonlocality caused by the extended nature of strings and is given by

$$
f=1-4 \mu \alpha_{(1)} \alpha_{(2)} \alpha_{(3)} K
$$


where $K$ is the well known expression in string field theory which is defined in terms of various infinite matrices associated with the familiar overlap condition for string interaction. For full details of the string field theory, we refer the reader to the Appendix B of the previous paper 2] and to the references cited therein and below. The 3-point interaction vertex takes just an equal-weight sum of the two previously constructed vertices which are compatible with supersymmery algebra:

$$
\left|H_{3}\right\rangle_{h} \equiv \frac{1}{2}\left(\left|H_{3}\right\rangle_{S V}+\left|H_{3}\right\rangle_{D}\right)
$$

where $\left|H_{3}\right\rangle_{S V}$ and $\left|H_{3}\right\rangle_{D}$ are those proposed in [6] and in [7, respectively. In particular, the prefactor of the part $\left|H_{3}\right\rangle_{D}$ manifestly takes the form of the energy difference $2 \mu \alpha_{1}$ while that of the part $\left|H_{3}\right\rangle_{S V}$ is the one obtained by a natural generalization of the familiar flat-space vertex.

Our convention for various parameters is as follows: $R^{4} / J_{1}^{2}\left(\alpha^{\prime}\right)^{2}=\lambda^{\prime}=1 /\left(\mu p_{1}^{+} \alpha^{\prime}\right)^{2}=$ $g_{Y M}^{2} N / J_{1}^{2}, g_{2}=J_{1}^{2} / N, \alpha_{(r)}=\alpha^{\prime} p_{r}^{+}, \mu\left|\alpha_{(r)}\right|=\alpha^{\prime} J_{r} / R^{2} \quad(r=1,2,3)$. Do not confuse the symbol $\alpha_{(1)}$, etc with the previously defined $\alpha_{1}$, etc as (2.6). Using these conventions, the large $\mu$ limit of $f$ is given as

$$
f \frac{J_{2} J_{3}}{J_{1}} \rightarrow \frac{J_{1}}{4 \pi \mu\left|\alpha_{(1)}\right|} .
$$

We assume $\alpha_{(1)}\left(=-\alpha_{(2)}-\alpha_{(3)}\right)<0, \alpha_{(2)}>0, \alpha_{(3)}>0$. Note that the mass parameter $\mu$ becomes a meaningful curvature parameter $\mu=1 / R$ as we identify the light-like momentum with the angular momentum by $\left|p_{r}^{+}\right|=J_{r} / R$, which is the correct one for comparing the action with flat-space form. The interaction term is of order $g_{s}$ for fixed $R$ and $p^{+}$ as it should be. The overall factor $\sqrt{J_{1} J_{2} J_{3}} / N$ is rewritten as $4 \pi g_{s}\left(\alpha^{\prime}\right)^{2} \sqrt{\left|p_{1}^{+} p_{2}^{+} p_{3}^{+}\right|} / R^{5 / 2}$ in terms of the usual string coupling constant $g_{s}$. This form indicates that the effective action in fact has a nontrivial curvature dependence.

The above normalization of the interaction vertex is fixed by matching the effective action in the supergravity sector. The string field is normalized such that the free action takes the standard Euclidean form

$$
\begin{gathered}
S_{2}=\int d \tau\left[\frac{1}{2}\left(\left\langle\bar{\psi}\left|\partial_{\tau}\right| \psi\right\rangle-\frac{1}{2}\left(\partial_{\tau}\langle\bar{\psi}|\right)|\psi\rangle+\left\langle\bar{\psi}\left|H_{2}\right| \psi\right\rangle\right],\right. \\
H_{2}^{(r)}=\frac{1}{\left|\alpha_{(r)}\right|} \sum_{n=-\infty}^{\infty} \omega_{n}^{(r)}\left(a_{n}^{(r) \dagger} a_{n}^{(r)}+b_{n}^{(r) \dagger} b_{n}^{(r)}\right) \quad \text { with } \quad \omega_{n}^{(r)}=\sqrt{n^{2}+\left(\mu \alpha_{(r)}\right)^{2}},
\end{gathered}
$$

where $\left(a_{n}^{(r)}, a_{n}^{(r) \dagger}\right)$ 's and $\left(b_{n}^{(r)}, b_{n}^{(r) \dagger}\right)$ 's represent 8-component bosonic and fermionic oscillators of $r$-th string. 
One of the most characteristic features of this string field theory is that for purely bosonic string states the (total) prefactor reduces to the special form which consists of only cos modes $a_{n}^{(r)}(n \geq 0)$ [8] and of only sin modes $a_{-n}^{(r)}(n>0)$ for scalar $(i=5 \sim 8)$ and vector $(i=1 \sim 4)$ impurities, respectively :

$$
\left|H_{3}\right\rangle_{h} \Rightarrow \sum_{r=1}^{3}\left(\sum_{i=5}^{8} \sum_{m=0}^{\infty} \frac{\omega_{m}^{(r)}}{\alpha_{(r)}} a_{m}^{(r) i \dagger} a_{m}^{(r) i}+\sum_{i=1}^{4} \sum_{m=1}^{\infty} \frac{\omega_{m}^{(r)}}{\alpha_{(r)}} a_{-m}^{(r) i \dagger} a_{-m}^{(r) i}\right)\left|E_{a}\right\rangle
$$

where $\left|E_{a}\right\rangle$ given as

$$
\left|E_{a}\right\rangle=\exp \left[-\frac{1}{2} \sum_{r, s=1}^{3} \sum_{m, n=-\infty}^{\infty} \alpha_{m}^{(r) \dagger} \tilde{N}_{m n}^{r s} \alpha_{n}^{(s) \dagger}\right]|0\rangle_{(1)(2)(3)}
$$

is the standard bosonic overlap vertex. Thus, $Z_{2}$ (or higher $\mathrm{SO}(8)$ symmetry) of the free string-field theory is completely violated by the interaction. The oscillators $\left(\alpha_{n}^{(r)}, \alpha_{n}^{(r) \dagger}, n \in\right.$ Z) in the exponential basis which directly corresponds to the standard convention for the BMN operators are related to the trigonometric basis by $(n>0)$

$$
\alpha_{0}=a_{0}, \quad \alpha_{n}=\frac{1}{\sqrt{2}}\left(a_{n}-i a_{-n}\right), \quad \alpha_{-n}=\frac{1}{\sqrt{2}}\left(a_{n}+i a_{-n}\right) .
$$

In our formalism, the BMN operators must have definite conformal dimensions to at least the first order in $g_{2}$ and to all orders with respect to $\lambda^{\prime}$. This means that for unprotected stringy BMN operators we have to take into account the effect of various operator mixing including double-trace operators [9] [10] to the leading approximation in $1 / N$ expansion in extracting the CFT coefficients $C_{123}$ on the gauge-theory side. In real life, we have to be satisfied by studies of leading $1 / \mu$-expansions at current stage of development, since computation of 3-point OPE coefficients at the order $g_{2} \lambda^{\prime}$ and beyond has not been carried out so far. Such a computation on the gauge-theory side in general requires two-loop calculations including operator mixing effects.

In [2], we have presented a general argument that the above relation must be correct for arbitrary impurity-preserving 3-point functions, by reinterpreting appropriately the previously known results [12] 7] for comparison between string field theory vertices and gauge-theory calculations in the leading large- $\mu$ expansion. We explicitly confirmed the

I As in [2], the minus sign on the exponential factor is due to our phase convention in defining the world-sheet oscillators. This is different from the standard one in the literature, but is necessary for matching between bulk and boundary. 
above relation for two-impurity processes including vector and spinor excitations . The peculiar correction factor $\left(f \frac{J_{2} J_{3}}{J_{1}}\right)^{-\left(\Delta_{2}+\Delta_{3}-\Delta_{1}\right) / 2} \Gamma\left(\frac{\Delta_{2}+\Delta_{3}-\Delta_{1}}{2}+1\right)$ appearing in the expression (2.3) can be neglected to the leading order in the $1 / \mu$-expansion for impurity-preserving processes. Thus, in these cases, the relation reduces to the one first conjectured in ref. 11] from a different viewpoint. Our previous work, however, clarified that this particular relation is valid only with our holographic string field theory vertex $\left|H_{3}\right\rangle_{h}$ which takes into account the operator mixing of gauge-theory operators by the above specific combination of two different prefactors.

When the impurities are not conserved, the correction factor plays a crucial role, as has already been shown by the construction in 2] for non-extremal correlators of chiral primary operators on the basis of the GKP-Witten relation. Our task is now to confirm this for unprotected stringy BMN operators by studying the large- $\mu$ limit. We can divide the impurity non-preserving 3-point interactions into two classes, class I and II, respectively, depending on $\alpha_{1}^{c l}=\left(\Delta_{2}^{c l}+\Delta_{3}^{c l}-\Delta_{1}^{c l}\right) / 2>0$ or $<0$ where $\Delta_{r}^{c l}$ denotes the classical conformal dimension, counting the number of fields and (spacetime) derivatives involved in each BMN operator. Because of the $\mathrm{SO}(4) \times \mathrm{SO}(4)$ symmetry, class II processes are possible only when we allow singlet representations for the external line 1.

\section{Class I non-singlet scalar impurities}

Let us start from considering a simple example of class I processes. We denote the directions of scalar excitations by $i, j, \ldots \in(5 \sim 8)$. The operator 2 is assumed to involve 4 scalar excitations in all different directions $i, j, k, \ell$ with world-sheet momenta $m,-m, p,-p$, respectively. The operators 3 and 1 are assumed to involve 2 scalar impurities in directions $k, \ell$ with momenta $q,-q$ and directions $i, j$ with momenta $n,-n$, respectively. The explicit forms are given, suppressing possible mixing terms with double-trace operators, as

$$
\begin{aligned}
& O_{(i, m ; j,-m ; k, p ; \ell,-p)}^{(2)}=\frac{1}{\sqrt{\left(J_{2}+3\right)^{3} N^{J_{2}+4}}} \sum_{a+b+c+d=J_{2}} \operatorname{Tr}\left[\phi_{i} Z^{a} \phi_{j} Z^{b} \phi_{k} Z^{c} \phi_{\ell} Z^{d}\right. \\
&\left.\times \quad \mathrm{e}^{\frac{2 \pi i}{\left(J_{2}+3\right)}[-a m+(a+b+1) p-(a+b+c+2) p]}+\text { permutations }\right]
\end{aligned}
$$


where the 'permutations' indicates the summation over all non-equivalent positioning of the impurities $(a, b, c, d \geq 0)$, and

$$
\begin{aligned}
& O_{(k, q ; \ell,-q)}^{(3)}=\frac{1}{\sqrt{\left(J_{3}+1\right) N^{J_{3}+2}}} \sum_{a=0}^{J_{3}} \operatorname{Tr}\left[\phi_{k} Z^{a} \phi_{\ell} Z^{J_{3}-a} \mathrm{e}^{-\frac{2 \pi i}{\left(J_{3}+1\right)} a q}\right], \\
& O_{(i, n ; j,-n)}^{(1)}=\frac{1}{\sqrt{\left(J_{1}+1\right) N^{J_{1}+2}}} \sum_{a=0}^{J_{1}} \operatorname{Tr}\left[\phi_{i} Z^{a} \phi_{j} Z^{J_{1}-a} \mathrm{e}^{-\frac{2 \pi i}{\left(J_{1}+1\right)} a n}\right] .
\end{aligned}
$$

The overall constants correspond to the normalization of scalar fields such that the free propagators are equal to (no summation over $i$ ) $\left\langle\phi_{i}\left(x_{1}\right) \phi_{i}\left(x_{2}\right)\right\rangle=\left\langle\bar{Z}\left(x_{1}\right) Z\left(x_{2}\right)\right\rangle=1 /\left|x_{12}\right|^{2}$. Note that the phases associated with nonzero momenta are determined only by relative (and oriented) distances among impurities. Here we have adopted the normalization constants and phase factors which are slightly different from those adopted in the recent literature. In the large $J$ limit, the difference between $J$ and $J+k-1$ with $k$ being the number of impurities is inconsequential for our leading order computations restricted to bosonic excitations. Therefore, in the following we will ignore this difference unless otherwise stated explicitly and use the more familiar convention by ignoring these shift of $J$, in order to save the space for mathematical expressions. However, for fermionic excitations it turns out that these shifts in the phases actually play an important role. Fermionic excitations will not, however, be treated in the present paper and be left for a separate work.

It is obvious that the gauge-theory correlator $\left\langle\bar{O}_{(i, n ; j,-n)}^{(1)} O_{(i, m ; j,-m ; k, p ; \ell,-p)}^{(2)} O_{(k, q ; \ell,-q)}^{(3)}\right\rangle$ in the leading planar approximation must necessarily involve contractions of impurity fields $\phi_{k}$ and $\phi_{\ell}$ between 2 and 3 , aside those between $1 \leftrightarrow 2$ and $1 \leftrightarrow 3$. This implies that to the lowest nontrival order in $g_{2}$ we can ignore mixing with double-trace operators in calculating the 3-point function, since the effect of the mixing terms becomes of higher order with respect to $\frac{1}{N}$, in contrast to the impurity preserving case where there is no contraction between 2 and 3 for the mixing contribution which corresponds to the topology of a product of two cylinders $(\times 1 / N)$. We then find by a straightforward free-field computation that the CFT coefficient is given by

$$
C_{123}=\frac{1}{N} \frac{1}{\sqrt{J_{1} J_{2}^{3} J_{3}}} \times 2 J_{1}^{2} \frac{\sin ^{2}(\pi y n)}{\pi^{2}\left(n-\frac{m}{y}\right)^{2}}=\frac{\sqrt{J_{1} J_{2} J_{3}}}{N} \frac{2}{J_{1}^{2} y^{2}(1-y)} \frac{\sin ^{2}(\pi y n)}{\pi^{2}\left(n-\frac{m}{y}\right)^{2}}
$$

with $y=J_{2} / J_{1}$. The first factor in the first equality comes from the overall normalization, and the second factor is the result of free-field contractions and of summation over the 
positioning of impurities. In particular, the factor 2 originates from the permutation of two contractions between operators 2 and 3. Since the contractions between 2 and 3 can occur in the planar limit only when the impurity fields $\phi_{k}$ and $\phi_{\ell}$ are adjacent to each other, they do not have any momentum dependence in the present large- $J$ limit. This is in general true for arbitrary configurations of momenta of such impurities at least for exchanges of bosonic fields, beyond the above special case.

Now let us turn to the corresponding calculation in string field theory. The string states are

$$
{ }_{(1)}\langle 0| \alpha_{n}^{i(1)} \alpha_{-n}^{j(1)}, \quad{ }_{(2)}\langle 0| \alpha_{m}^{i(2)} \alpha_{-m}^{j(2)} \alpha_{p}^{k(2)} \alpha_{-p}^{\ell(2)}, \quad{ }_{(3)}\langle 0| \alpha_{q}^{k(3)} \alpha_{-q}^{\ell(3)},
$$

respectively. The relevant part of the interaction vertex takes the form

$$
\left|H_{3}\right\rangle_{h} \Rightarrow P_{123} \exp \left[-\mathcal{N}^{12}-\mathcal{N}^{23}\right]|0\rangle_{(1),(2),(3)}
$$

where the prefactor is

$$
\begin{gathered}
P_{123}=\frac{\omega_{m}^{(2)}}{\alpha_{(2)}}\left(2+\alpha_{m}^{(2) \dagger} \alpha_{-m}^{(2)}+\alpha_{-m}^{(2) \dagger} \alpha_{m}^{(2)}\right)+\frac{\omega_{p}^{(2)}}{\alpha_{(2)}}\left(2+\alpha_{p}^{(2) \dagger} \alpha_{-p}^{(2)}+\alpha_{-p}^{(2) \dagger} \alpha_{p}^{(2)}\right) \\
+\frac{\omega_{q}^{(3)}}{\alpha_{(3)}}\left(2+\alpha_{q}^{(3) \dagger} \alpha_{-q}^{(3)}+\alpha_{-q}^{(3) \dagger} \alpha_{q}^{(3)}\right)-\frac{\omega_{n}^{(1)}}{\left|\alpha_{(1)}\right|}\left(2+\alpha_{n}^{(1) \dagger} \alpha_{-n}^{(1)}+\alpha_{-n}^{(1) \dagger} \alpha_{n}^{(1)}\right)
\end{gathered}
$$

and $\mathcal{N}^{r s}$ are expressed in terms of the Neumann functions in the exponential basis as

$$
\begin{aligned}
\mathcal{N}^{12} & =\tilde{N}_{n m}^{12}\left(\alpha_{n}^{(1) \dagger} \alpha_{m}^{(2) \dagger}+\alpha_{-n}^{(1) \dagger} \alpha_{-m}^{(2) \dagger}\right)+\tilde{N}_{-n m}^{12}\left(\alpha_{-n}^{(1) \dagger} \alpha_{m}^{(2) \dagger}+\alpha_{n}^{(1) \dagger} \alpha_{-m}^{(2) \dagger}\right) \\
& +\tilde{N}_{n p}^{12}\left(\alpha_{n}^{(1) \dagger} \alpha_{p}^{(2) \dagger}+\alpha_{-n}^{(1) \dagger} \alpha_{-p}^{(2) \dagger}\right)+\tilde{N}_{-n p}^{12}\left(\alpha_{-n}^{(1) \dagger} \alpha_{p}^{(2) \dagger}+\alpha_{n}^{(1) \dagger} \alpha_{-p}^{(2) \dagger}\right), \\
\mathcal{N}^{23} & =\tilde{N}_{p q}^{23}\left(\alpha_{p}^{(2) \dagger} \alpha_{q}^{(3) \dagger}+\alpha_{-p}^{(2) \dagger} \alpha_{-q}^{(3) \dagger}\right)+\tilde{N}_{-p q}^{23}\left(\alpha_{-p}^{(2) \dagger} \alpha_{q}^{(3) \dagger}+\alpha_{p}^{(2) \dagger} \alpha_{-q}^{(3) \dagger}\right) .
\end{aligned}
$$

Note that there is no contraction between $1 \leftrightarrow 3$.

In the leading large $\mu$ limit we can simply replace the energy factor $\omega_{n}^{(r)} /\left|\alpha_{(r)}\right|$ by $\mu$ for class I processes, since there is no singularity in the multiplying factor (denoted by $G$ ) of the holographic relation

$$
G \equiv\left(f \frac{J_{2} J_{3}}{J_{1}}\right)^{-\left(\Delta_{2}+\Delta_{3}-\Delta_{1}\right) / 2} \frac{\Gamma\left(\frac{\Delta_{2}+\Delta_{3}-\Delta_{1}}{2}+1\right)}{\mu\left(\Delta_{2}+\Delta_{3}-\Delta_{1}\right)} \sim \frac{\alpha_{1}^{c l} !}{2 \mu \alpha_{1}^{c l}}\left(\frac{J_{1}}{4 \pi \mu\left|\alpha_{(1)}\right|}\right)^{-\alpha_{1}^{c l}},
$$

with $\alpha_{1}=\left(\Delta_{2}+\Delta_{3}-\Delta_{1}\right) / 2 \sim \alpha_{1}^{c l}+O\left(1 / \mu^{2}\right)$. In the present example, $\alpha_{1}^{c l}=2$. Using the properties of Neumann functions such as $\tilde{N}_{n m}^{r s}=\tilde{N}_{-n-m}^{r s}, \tilde{N}_{-n m}^{r s}=\tilde{N}_{n-m}^{r s}$ for $m, n>0$, we 
can easily see that the contributions from two parts of the prefactor involving $\alpha_{m}^{(2) \dagger} \alpha_{-m}^{(2)}+$ $\alpha_{-m}^{(2) \dagger} \alpha_{m}^{(2)}$ and $\alpha_{n}^{(1) \dagger} \alpha_{-n}^{(1)}+\alpha_{-n}^{(1) \dagger} \alpha_{n}^{(1)}$, respectively, cancel against each other. This cancellation corresponds to the fact that two terms $\left|H_{3}\right\rangle_{S V}$ and $\left|H_{3}\right\rangle_{D}$ have equal contributions in this case. The situation is in contrast to the impurity-preserving sector where they play different roles (roughly speaking, 'bare' interaction and mixing effect, respectively), owing to the existence of the singularity in the factor $G$. Furthermore, using the property that the large $\mu$ limit of $\left|\tilde{N}_{p q}^{23}\right|$ is independent of the momenta, we find that the matrix element of the interaction vertex is equal to

$$
4 \mu\left(\tilde{N}_{n m}^{12} \tilde{N}_{p q}^{23}\right)^{2}
$$

Using the explicit expressions of Neumann functions (see the Appendix for a summary) in the leading large $\mu$ limit,

$$
\tilde{N}_{n m}^{12}=\frac{(-1)^{m+n+1} \sin (\pi n y)}{\pi \sqrt{y}\left(n-\frac{m}{y}\right)}, \quad \tilde{N}_{p q}^{23}=\frac{(-1)^{p+1}}{4 \pi \mu\left|\alpha_{(1)}\right| \sqrt{y(1-y)}},
$$

we find that the CFT coefficient (3.4) from gauge-theory side precisely matches the string interaction vertex with the holographic relation $(2.1) \sim(2.3)$.

It is not difficult to extend the above result to a more general case of scalar impurities. In this section, we restrict ourselves to operators without any singlet representation with respect to $\mathrm{O}(4)$ group of rotations of scalar directions. More precisely, the scalar impurities $\phi_{i_{1}}, \phi_{i_{2}}, \ldots$ are contracted to 'polarization' tensors $C_{i_{1} i_{2} \ldots}^{I}$ which are traceless with respect to arbitrary pair of the $\mathrm{O}(4)$ indices $\left(i_{1}, i_{2}, \ldots\right)$ for each conformal BMN operator in an appropriate irreducible representation of $\mathrm{SO}(4)$ :

$$
\begin{gathered}
O_{\left(p_{1}, p_{2}, \ldots\right)}^{I}=\frac{C_{i_{1} i_{2} \cdots}^{I}}{\sqrt{J^{k-1} N^{J+k}}} \sum_{a_{1}+a_{2}+\cdots=J} \operatorname{Tr}\left[\phi_{i_{1}} Z^{a_{1}} \phi_{i_{2}} Z^{a_{2}} \cdots \mathrm{e}^{2 \pi i\left(a_{1} p_{2}+a_{2} p_{3}+\cdots\right) / J}\right. \\
+ \text { permutations }],
\end{gathered}
$$

where $k(\geq 1)$ is the number of impurities and as above we have to sum over non-equivalent positioning of impurities." The traceless condition allows us to ignore the mixing of the pairs of operators $Z$ and $\bar{Z}$ which would be needed [13] for defining operators with definite

"Note that we have changed the notations slightly from ref. [2]. For instance, $\tilde{k} \rightarrow k$. The convention on the summation over 'permutations' (for sugra modes in particular) is also changed. In the present paper, 'non-equivalent' permutations exclude those which correspond to cyclic permutations, while in [2] they were not excluded and hence the normalization constants had an additional power of $1 / J$. 
conformal dimensions even if the mixing with double-trace operators can be ignored. If we consider higher orders in $g_{\mathrm{YM}}$, mixing between purely bosonic operators with antisymmetrized scalar indices (or mixed scalar and vector indices) and those involving fermion impurities are expected to play important roles. In this paper, we ignore such complications by restricting ourselves to the leading order effect in the large- $\mu$ expansion on 3-point functions. The symbol $I$ of the polarization tensor indicates collectively the configurations of both momenta and $\mathrm{SO}(4)$ representation. Its normalization is most conveniently expressed using oscillators $a_{p}^{(1) \dagger}, a_{p}^{(1)}$, etc of strings, as

$$
\begin{gathered}
{ }_{(1)}\left\langle\left. C^{I_{1}}\right|_{(2)}\left\langle C^{I_{2}}\left|\exp \left[\sum_{p=-\infty}^{\infty} \alpha_{p}^{(1) \dagger} \alpha_{p}^{(2) \dagger}\right]\right| 0\right\rangle_{(1)(2)}=\delta^{I_{1} I_{2}}\right. \\
{ }_{(1)}\left\langle C^{I_{1}}\right|={ }_{(1)}\langle 0| C_{i_{1} i_{2} \ldots}^{I_{1}} \alpha_{p_{1}}^{i_{1}(1)} \alpha_{p_{2}}^{i_{2}(1)} \ldots \text { etc }
\end{gathered}
$$

where the $\mathrm{SO}(4)$ indices on the exponential is suppressed. According to this convention, various symmetry factors associated with the symmetry property of the $\mathrm{SO}(4)$ indices are absorbed in the normalization of polarization tensors themselves. This representation naturally takes into account the summation over all allowed contractions with equal weights corresponding to those of free-gauge theory. Of course, the momenta must satisfy the level matching condition

$$
\sum_{i=1}^{k} p_{k}=0 .
$$

Suppose the $r$-th operator has $k_{r}$ impurities. Then, the number of contractions between $r=2$ and $r=3$ is equal to

$$
\alpha_{1}^{c l}=\left(k_{2}+k_{3}-k_{1}\right) / 2 .
$$

Now, in computing the gauge-theory correlators for this general type of operators, we note the following properties, all of which already appeared in the above example.

1. We can ignore operator mixing with double-trace operators, since the mixing contributions are always of higher order in the $1 / N$-expansion because of the existence of nonzero number of 2-3 contractions. In the absence of the mixing with doubeltrace operators, the CFT coefficients to the leading order in $1 / \mu$ can be determined entirely from the free-field contractions which give the correct leading order form of the spacetime factor of conformal 3-point functions. 


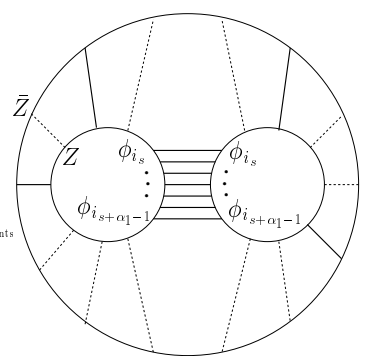

Figure 1: The dotted lines represent $Z-\bar{Z}$ contractions, while real lines represent contractions of scalar fields $\phi_{i}$ 's. The outer circle denotes the trace of operator product in the line 1 and two inner circles the traces of operator products in the line 2 and 3 , respectively.

2. In the planar limit, the contractions between 2 and 3 must belong to a single group of adjacent products of the impurity $\phi_{i}$ fields in which no $Z$ field is contained, since all $Z$ fields in the operators 2 and 3 must be contracted with $\bar{Z}$ fields in the operator 1 and hence would lead to a nonplanar contribution otherwise. In other words, in the sum over $a_{i}$ in the definition (3.11), such contractions are possible only when $a_{i_{s}}=a_{i_{s+1}}=\cdots=a_{i_{s+\alpha_{1}-1}}=0$ with some $s$ for a consecutive set of impurities $\left(\phi_{i_{s}}, \phi_{i_{s+1}}, \ldots, \phi_{i_{s+\alpha_{1}-1}}\right)$. See fig. 1 .

3. By the cyclic symmetry of Tr operation and the level-matching condition, we can always choose $s=1$. This means that we can replace the momentum-dependent exponential by one for these impurities in the large- $J$ limit. Therefore, the $2 \leftrightarrow 3$ contractions do not give any momentum dependent factor.

Using these properties, the 3-point correlation function is computed as follows. First, the overall constant factor is

$$
\alpha_{1}^{c l} ! \frac{N^{J_{1}+k_{1}+\alpha_{1}^{c l}-1}}{\sqrt{\prod_{i=1}^{3} J_{i}^{k_{i}-1} N^{J_{i}+k_{i}}}}=\frac{\sqrt{J_{1} J_{2} J_{3}}}{N} \alpha_{1}^{c l} ! J_{1}^{-\left(k_{1}+k_{2}+k_{3}\right) / 2}\left(\frac{J_{2}}{J_{1}}\right)^{-k_{2} / 2}\left(\frac{J_{3}}{J_{1}}\right)^{-k_{3} / 2}
$$

which can also be written as

$$
\frac{\sqrt{J_{1} J_{2} J_{3}}}{N} \times J_{1}^{-k_{1}}\left(\frac{J_{2}}{J_{1}}\right)^{-\bar{k}_{2} / 2}\left(\frac{J_{3}}{J_{1}}\right)^{-\bar{k}_{3} / 2} \times \alpha_{1}^{c l} ! J_{1}^{-\alpha_{1}^{c l}} \times(\sqrt{y(1-y)})^{-\alpha_{1}^{c l}}
$$

where $\bar{k}_{2}=k_{2}-\alpha_{1}^{c l}$ and $\bar{k}_{3}=k_{3}-\alpha_{1}^{c l}$ represent the number of impurities in the operators 2 and 3 , respectively, which contract with those of the operator 1 . Note that the factor 
$\alpha_{1}^{c l}$ ! comes from the permutations of the ordering of impurities contracting between 2 and 3.

Secondly, each single contraction with momenta $p_{i}^{(1)}$ and $p_{i}^{(2)}$ respectively between 1 and 2 after the summation over the permutation of their positioning gives the factor (suppressing the spacetime dependent factor $1 /\left|\vec{x}_{12}\right|^{2}$ )

$$
\sum_{a=0}^{J_{2}} \exp \left[2 \pi i a\left(\frac{p_{i}^{(2)}}{J_{2}}-\frac{p_{i}^{(1)}}{J_{1}}\right)\right] \rightarrow J_{1} \mathrm{e}^{-\pi i y p_{i}^{(1)}} \frac{\sin \pi y p_{i}^{(1)}}{\pi\left(p_{i}^{(1)}-\frac{p_{i}^{(2)}}{y}\right)}
$$

in the large $J$ limit, which is expressed in terms of Neumann function as

$$
J_{1} \sqrt{y}(-1)^{p_{i}^{(1)}+p_{i}^{(2)}+1} \mathrm{e}^{-\pi i y p_{i}^{(1)}} \tilde{N}_{p_{i}^{(1)} p_{i}^{(2)}}
$$

Here the Kronecker delta of the corresponding $\mathrm{SO}(4)$ indices is suppressed. Similarly, each single contraction with momenta $q_{i}^{(1)}$ and $q_{i}^{(3)}$ respectively between 1 and 3 gives

$$
\sum_{a=0}^{J_{3}} \exp \left[2 \pi i a\left(-\frac{q_{i}^{(3)}}{J_{3}}+\frac{q_{i}^{(1)}}{J_{1}}\right)\right] \rightarrow-J_{1} \sqrt{1-y} \mathrm{e}^{\pi i(1-y) q_{i}^{(1)}} \tilde{N}_{q_{i}^{(1)} q_{i}^{(3)}}^{13}
$$

On the other hand, each contraction with momenta $r_{i}^{(2)}$ and $r_{i}^{(3)}$ respectively between 2 and 3 gives factor 1 (again apart from the spacetime factor) which is replaced by the corresponding Neumann functions using the identity

$$
1=(-1)^{r_{i}^{(2)}+1} 4 \pi \mu\left|\alpha_{(1)}\right| \sqrt{y(1-y)} \tilde{N}_{r_{i}^{(2)} r_{i}^{(3)}}^{23}
$$

Collecting all these factors together, we conclude that the CFT coefficient is given as

$$
\begin{gathered}
C_{123}=\left(C^{I_{1}} C^{I_{2}} C^{I_{3}}\right)(-1)^{\alpha_{1}^{c l}+\alpha_{2}^{c l}+\alpha_{3}^{c l}} \frac{\sqrt{J_{1} J_{2} J_{3}}}{N} \times J_{1}^{-k_{1}}\left(\frac{J_{2}}{J_{1}}\right)^{-\bar{k}_{2} / 2}\left(\frac{J_{3}}{J_{1}}\right)^{-\bar{k}_{3} / 2} \times \alpha_{1}^{c l} ! J_{1}^{-\alpha_{1}^{c l}} \\
\times(\sqrt{y(1-y)})^{-\alpha_{1}}\left(\prod_{12} J_{1} \sqrt{y} \tilde{N}_{p_{i}^{(1)} p_{i}^{(2)}}^{12}\right)\left(\prod_{13} J_{1} \sqrt{1-y} \tilde{N}_{q_{i}^{(1)}}^{13} q_{i}^{(3)}\right) \\
\times\left(\prod_{23} 4 \pi \mu\left|\alpha_{(1)}\right| \sqrt{y(1-y)} \tilde{N}_{r_{i}^{(2)} r_{i}^{(3)}}^{23}\right) \\
=\left(C^{I_{1}} C^{I_{2}} C^{I_{3}}\right)(-1)^{\alpha_{1}^{c l}+\alpha_{2}^{c l}+\alpha_{3}^{c l}} \frac{\sqrt{J_{1} J_{2} J_{3}}}{N} \alpha_{1} !\left(\frac{J_{1}}{4 \pi \mu\left|\alpha_{(1)}^{c l}\right|}\right)^{-\alpha_{1}^{c l}} \\
\times\left(\prod_{12} \tilde{N}_{p_{i}^{(1)} p_{i}^{(2)}}^{12}\right)\left(\prod_{13} \tilde{N}_{q_{i}^{(1)} q_{i}^{(3)}}^{13}\right)\left(\prod_{23} \tilde{N}_{r_{i}^{(2)} r_{i}^{(3)}}^{23}\right)
\end{gathered}
$$


where $\prod_{r s}$ denotes the product over the contractions and the symbol $\left(C^{I_{1}} C^{I_{2}} C^{I_{3}}\right)$ denotes the part of $\mathrm{SO}(4)$ contractions of polarization tensors associated with the free-field contractions. We note that the phase factors of the expressions $(3.17) \sim(3.19)$ cancel using the level-matching condition and that in terms of string notation, the following equality

$$
\begin{gathered}
\left(C^{I_{1}} C^{I_{2}} C^{I_{3}}\right)(-1)^{\alpha_{1}^{c l}+\alpha_{2}^{c l}+\alpha_{3}^{c l}}\left(\prod_{12} \tilde{N}_{p_{i}^{(1)} p_{i}^{(2)}}^{12}\right)\left(\prod_{13} \tilde{N}_{q_{i}^{(1)} q_{i}^{(3)}}^{13}\right)\left(\prod_{23} \tilde{N}_{r_{i}^{(2)} r_{i}^{(3)}}^{23}\right) \\
={ }_{(1)}\left\langleC ^ { I _ { 1 } } | _ { ( 2 ) } \left\langle\left. C^{I_{2}}\right|_{(3)}\left\langle C^{I_{3}}|| E_{a}\right\rangle\right.\right.
\end{gathered}
$$

is valid.

Let us next confirm that this result matches the string field theory through our holographic relation. Since we have already expressed a main part of the CFT coefficient obtained on the gauge-theory side in terms of string states, only nontrivial part for this task is to examine the prefactor. In the large $\mu$-limit, it takes the form

$$
\begin{gathered}
P_{123}=\mu\left(\alpha_{0}^{(2) \dagger} \alpha_{0}^{(2)}+\alpha_{0}^{(3) \dagger} \alpha_{0}^{(3)}-\alpha_{0}^{(1) \dagger} \alpha_{0}^{(1)}\right) \\
+\frac{\mu}{2} \sum_{m=1}^{\infty}\left(\alpha_{m}^{(2) \dagger} \alpha_{m}^{(2)}+\alpha_{m}^{(3) \dagger} \alpha_{m}^{(3)}-\alpha_{m}^{(1) \dagger} \alpha_{m}^{(1)}+[m \rightarrow-m]\right) \\
+\frac{\mu}{2} \sum_{m=1}^{\infty}\left(\alpha_{m}^{(2) \dagger} \alpha_{-m}^{(2)}+\alpha_{m}^{(3) \dagger} \alpha_{-m}^{(3)}-\alpha_{m}^{(1) \dagger} \alpha_{-m}^{(1)}+[m \rightarrow-m]\right)
\end{gathered}
$$

Obviously, the contributions from the first and second lines is simply determined by counting the difference of the numbers of impurities in the process $2+3 \rightarrow 1$. Furthermore, in the third line, the impurity preserving part corresponding to contractions $1 \leftrightarrow 2$ and $1 \leftrightarrow 3$ cancels due to the equalities $\tilde{N}_{-n m}^{r s}=\tilde{N}_{n-m}^{r s}, \tilde{N}_{n m}^{r s}=\tilde{N}_{-n-m}^{r s}$. Then, the property that $\tilde{N}_{m n}^{23}$ is independent of the signs of momenta $(m, n)$ shows that the contribution of the third term is $\mu \alpha_{1}^{c l}$. Thus all together the total contribution of the prefactor is $2 \mu \alpha_{1}^{c l}$. But this is just canceled by the denominator factor $1 / \mu\left(\Delta_{2}+\Delta_{3}-\Delta_{1}\right)$ of the holographic relation. Using (3.21), it is now clear that the CFT coefficient precisely satisfies our holographic relation $(2.1) \sim(2.3)$ including the sign and numerical factors.

\section{Class I non-singlet vector impurities}

In this section, we extend the result of the previous section to the situation where (nonsinglet) vector excitations are involved. A vector excitation of momentum $p$ at the position $a$ corresponds to the insertion of a derivative $\mathrm{e}^{2 \pi i a p / J} D_{i}(i \in(1 \sim 4))$ in the trace (3.11). 
We assume here that only traceless part are involved with respect to the vector $\mathrm{SO}(4)$ indices too. So, the vector $\mathrm{SO}(4)$ indices which will be mostly suppressed below should be understood to be contracted with traceless polarization tensors similarly as in the previous section. As we have argued in detail in 2] on the basis of the GKP-Witten relation, the derivatives must then be computed by assuming the following form for the variation of the distance function $\left|\vec{x}_{r s}\right|$ under the shift of the spacetime coordinates $\vec{x}_{r} \rightarrow \vec{x}+\delta \vec{x}_{r}$,

$$
\frac{1}{\left|\vec{x}_{r s}\right|^{2}} \rightarrow \frac{1}{\left(\vec{x}_{r}-\vec{x}_{s}\right)^{2}-2 \delta \vec{x}_{r} \cdot \delta \vec{x}_{s}}
$$

in order to be consistent with the $\mathrm{SO}(4)$ symmetry and orthogonality of vector states. The invariants such as $(\delta \vec{x})^{2}$ can be ignored because of the traceless condition.

This allows us to treat the pairs of vector indices almost as those of additional virtual scalar indices. Namely, the pair of derivatives of the spacetime factor of a free propagator can contribute in the form

$$
\sum_{s} \mathrm{e}^{2 \pi i a\left(\frac{p^{(r)}}{J_{r}} \pm \frac{q^{(s)}}{J_{s}}\right)} \partial_{i}^{(r)} \partial_{j}^{(s)} \frac{1}{\left|\vec{x}_{r s}\right|^{2}} \sim \frac{2}{\left|\vec{x}_{r s}\right|^{4}} \delta_{i j} \tilde{N}_{p^{(r)}, \pm q^{(s)}}^{r s} \times \text { phase factor }
$$

where the phase factor depending on $(r, s)$ is the same as for the case of scalars. Thus a pair of vector derivatives yields essentially the same factor as in the case of contractions of scalar impurities, except for the factor 2 which is absorbed in the normalization, provided that they act on the free propagator. This implies that the derivatives acting on $1 \leftrightarrow 2$ and $1 \leftrightarrow 3$ contractions give the same factors on both sides of gauge theory and string field theory. In other words, the holographic relation is satisfied as it stands when vector impurities are conserved for arbitrary impurity non-preserving processes in which impurity non-preserving contractions between 2 and 3 only occur for scalar excitations.

Therefore, in the rest of this section, it is sufficient to concentrate to the case where all vector impurities contribute to $(2 \leftrightarrow 3)$ contractions. Suppose first that we add $\beta_{1}^{c l}$ such derivatives for a process with nonzero scalar exchange in the $2-3$ channel $\left(\alpha_{1}^{c l}>0\right)$. Since the spacetime factor $1 /\left|\vec{x}_{23}\right|^{2 \alpha_{1}^{c l}}$ only comes from a single group of adjacent scalar impurities, the derivatives act as

$$
\frac{1}{\left(2 J_{2}\right)^{\beta_{1}^{c l} / 2}\left(2 J_{3}\right)^{\beta_{1}^{c l} / 2}} \times \frac{1}{\left(\beta_{1}^{c l}\right) !}\left(\partial^{(2)}\right)^{\beta_{1}^{c l}}\left(\partial^{(3)}\right)^{\beta_{1}^{c l}} \frac{1}{\left|\vec{x}_{23}\right|^{2 \alpha_{1}^{c l}}}
$$

where the first factor comes from the normalization constant associated with the vector excitations. For notational simplicity, we suppress the $\mathrm{SO}(4)$ vector indices. The denominator factor $1 /\left(\beta_{1}^{c l}\right)$ ! is the symmetry factor to cancel the over-counting. This is equal 
to

$$
\begin{gathered}
J_{1}^{-\beta_{1}^{c l}}\left(\frac{J_{2}}{J_{1}}\right)^{-\beta_{1}^{c l} / 2}\left(\frac{J_{3}}{J_{1}}\right)^{-\beta_{1}^{c l} / 2} \times \frac{\Gamma\left(\alpha_{1}^{c l}+\beta_{1}^{c l}\right)}{\Gamma\left(\alpha_{1}^{c l}\right)} \frac{1}{\left|\vec{x}_{23}\right|^{2\left(\alpha_{1}^{c l}+\beta_{1}^{c l}\right)}} \\
=J_{1}^{-\beta_{1}^{c l}}(\sqrt{y(1-y)})^{-\beta_{1}^{c l}} \frac{\Gamma\left(\alpha_{1}^{c l}+\beta_{1}^{c l}+1\right)}{\Gamma\left(\alpha_{1}^{c l}+1\right)} \frac{2 \alpha_{1}^{c l}}{2\left(\alpha_{1}^{c l}+\beta_{1}^{c l}\right)} \frac{1}{\left|\vec{x}_{23}\right|^{2\left(\alpha_{1}^{c l}+\beta_{1}^{c l}\right)}} .
\end{gathered}
$$

As in the scalar case, we further multiply the factor

$$
1=(-1)^{r_{i}^{(2)}+1} 4 \pi \mu\left|\alpha_{(1)}\right| \sqrt{y(1-y)} \tilde{N}_{r_{i}^{(2)} r_{i}^{(3)}}^{23}
$$

corresponding to each pair of derivatives $\partial^{(2)} \partial^{(3)}$. By this procedure, the power of $y(1-y)$ is canceled and replaced by the Neumann functions for $(2-3)$ channel, leaving us the correction factor $\left(\frac{J_{1}}{4 \pi \mu \mid \alpha_{(1)}^{c l}}\right)^{-\beta_{1}^{c l}}$. Furthermore, the factor $\frac{\Gamma\left(\alpha_{1}^{c l}+\beta_{1}^{c l}+1\right)}{\Gamma\left(\alpha_{1}^{c l}+1\right)} \frac{2 \alpha_{1}^{c l}}{2\left(\alpha_{1}^{c l}+\beta_{1}^{c l}\right)}$ is just the one required for modifying the energy factor in the holographic relation as

$$
\frac{\Gamma\left(\alpha_{1}^{c l}+1\right)}{2 \alpha_{1}^{c l}} \rightarrow \frac{\Gamma\left(\alpha_{1}^{c l}+\beta_{1}^{c l}+1\right)}{2\left(\alpha_{1}^{c l}+\beta_{1}^{c l}\right)}
$$

corresponding to the shift of $\Delta_{2}+\Delta_{3}-\Delta_{1}$ due to the addition of vector impurities in the $(2-3)$ channel.

Turning now to the string side, only difference for vector excitations from the purely scalar case is that the contribution of vector modes to the prefactor consists only of sin modes,

$$
\begin{gathered}
\frac{\mu}{2} \sum_{m=1}^{\infty}\left(\alpha_{m}^{(2) \dagger} \alpha_{m}^{(2)}+\alpha_{m}^{(3) \dagger} \alpha_{m}^{(3)}-\alpha_{m}^{(1) \dagger} \alpha_{m}^{(1)}+[m \rightarrow-m]\right) \\
-\frac{\mu}{2} \sum_{m=1}^{\infty}\left(\alpha_{m}^{(2) \dagger} \alpha_{-m}^{(2)}+\alpha_{m}^{(3) \dagger} \alpha_{-m}^{(3)}-\alpha_{m}^{(1) \dagger} \alpha_{-m}^{(1)}+[m \rightarrow-m]\right) .
\end{gathered}
$$

Then, the independence of $(2-3)$ Neumann functions on the momentum (except for the sign factor) shows that the vector contribution in this channel is zero due to cancellation between the first and the second line in this expression. Hence, it does not contribute at all to the modification of the 3-point function. Also it is easy to check that the sign and phase factors exactly match using level matching condition, in the same way as purely scalar case of the previous section. Thus we can conclude that the holographic relation is precisely satisfied.

The case with $\alpha_{1}^{c l}=0$ requires a separate consideration. In this case, there is no direct contraction for scalar fields in the $(2-3)$ channel. Also the correction factor before taking derivatives is singular in the large $\mu$-limit, and we have to take into account mixing with 
double-trace operators. The space-time dependence come from the order $\lambda^{\prime}$ contributions to the anomalous dimensions. Let us proceed inductively, starting from $\beta_{1}^{c l}=1$. We have to consider

$$
\frac{1}{\left(2 J_{2}\right)^{1 / 2}\left(2 J_{3}\right)^{1 / 2}} \times \partial^{(2)} \partial^{(3)} \frac{1}{\left|\vec{x}_{23}\right|^{2 \alpha_{1}}} \sim\left(J_{2} J_{3}\right)^{-1 / 2} \alpha_{1} \frac{1}{\left|\vec{x}_{23}\right|^{2}}
$$

with $\alpha_{1} \sim O\left(\left(\mu \alpha_{(1)}\right)^{-2}\right)$. The factor $\alpha_{1}$ is precisely the necessary factor which modifies the singular energy factor into non-singular one corresponding to nonzero $\beta_{1}^{c l}$,

$$
\frac{\Gamma\left(\alpha_{1}+1\right)}{2 \alpha_{1}} \sim \frac{\Gamma\left(\alpha_{1}^{c l}+1\right)}{2 \alpha_{1}} \rightarrow \alpha_{1} \times \frac{\Gamma\left(\alpha_{1}^{c l}+1\right)}{2 \alpha_{1}}=\frac{\Gamma\left(\alpha_{1}^{c l}+\beta_{1}^{c l}+1\right)}{2\left(\alpha_{1}^{c l}+\beta_{1}^{c l}\right)}=\frac{1}{2},
$$

with $\beta_{1}^{c l}=1$ and $\alpha_{1}^{c l}=0$, and the power $\left(J_{2} J_{3}\right)^{-1 / 2}$ plays the same role generating the $(2-3)$ Neumann functions as in the above case with nonzero $\alpha_{1}^{c l}$. Since there does not occur any correction factor from the vector prefactor on the string side, this proves the validity of the holographic relation. Note that in this special case the mixing with double-trace operator for the operator 1 is effective on the gauge-theory side, but on the string side the $(2-3)$ Neumann functions contribute the same common factor for both $\left|H_{3}\right\rangle_{D}$ and $\left|H_{3}\right\rangle_{S V}$. Once the spacetime factor $1 /\left|\vec{x}_{23}\right|^{2}$ is generated correspondingly to a single vector exchange as above, we can apply the same argument inductively at each time of adding one vector exchange between 2 and 3 as we have discussed for the case starting from nonzero $\alpha_{1}^{c l}$. This completes our argument for the holographic relation for non-singlet vector impurities.

\section{Singlet operators and class II processes}

All previous arguments are restricted to cases where there is no trace part in the external lines. When we allow trace part for the $\mathrm{SO}(4)$ representation, the situation becomes more cumbersome and it is not easy to make treatments in a general way because of various possible mixings among different configurations of operator products even at the level of single trace operators. It is also important that the inclusion of singlet opens the possibility of class II processes. In the present paper, we study several typical cases in order to convince ourselves that our holographic relation must be valid in such cases too. In particular, we will see that various characteristic structures of the CFT coefficients depending differently on scalar and vector excitations are nicely captured by the interplay between the factor $G$ in the holographic relation and the very specific prefactor of our string-interaction vertex. 


\subsection{Class I with scalar singlets}

The simplest (normalized) BMN operator with singlet representation for scalar excitation [13] is

$$
O_{s s(p ;-p)}=\frac{1}{2 \sqrt{J N^{J+2}}} \operatorname{Tr}\left[\sum_{a=0}^{J} \phi_{i} Z^{a} \phi_{i} Z^{J-a} \mathrm{e}^{-\frac{2 \pi i}{J} a p}-4 \bar{Z} Z^{J+1}\right]
$$

where the scalar index $i$ is summed over from $i=5$ to 8 and $p \neq 0$. When $p=0$, the normalization constant should be replaced by $\frac{1}{2 \sqrt{2 J N^{J+2}}}$. The necessity of the mixing of the second term can be easily understood when $p=0$ : In order for this operator to be BPS, it must be symmetric and traceless with respect to $\mathrm{SO}(6)$ symmetry. The role of the second term is to meet this requirement by completing the vanishing $\mathrm{SO}(6)$ trace from the $Z-\bar{Z}$ directions.

Let us start from the case where the operator 1 and 3 are in the ground state,

$$
O^{(1)}=\frac{1}{\sqrt{J_{1} N^{J_{1}}}} \operatorname{Tr}\left[Z^{J_{1}}\right], \quad O^{(3)}=\frac{1}{\sqrt{J_{3} N^{J_{3}}}} \operatorname{Tr}\left[Z^{J_{3}}\right],
$$

and the operator 2 is the above singlet-state operator,

$$
O_{s(p ;-p)}^{(2)}=\frac{1}{2 \sqrt{J_{2} N^{J_{2}+2}}} \operatorname{Tr}\left[\sum_{a=0}^{J_{2}} \phi_{i} Z^{a} \phi_{i} Z^{J_{2}-a} \mathrm{e}^{-\frac{2 \pi i}{J_{2}} a p}-4 \bar{Z} Z^{J_{2}+1}\right] .
$$

On the gauge-theory side, the first term of (5.3) has no contribution in the leading order in the perturbation theory. The second term can contribute in the leading order if and only if one of the $\bar{Z}$ in $O_{s(p ;-p)}^{(2)}$ is contracted with one of $Z$ in $O^{(3)}$ as in fig. 2. The mixing with double-trace operator can again be ignored. Thus, apart from the spacetime factor (which takes the correct form), the CFT coefficient is given as

$$
C_{123}=-4 \times \frac{1}{2 \sqrt{J_{2} N^{J_{2}+2}}} \times \frac{1}{\sqrt{J_{1} N^{J_{1}} J_{3} N^{J_{3}}}} J_{3} J_{1} N^{J_{1}}=-2 \frac{\sqrt{J_{1} J_{2} J_{3}}}{N} \frac{1}{J_{2}} .
$$

On the string side, the state corresponding to the operator 2 is

$$
\text { (2) }\langle 0| \frac{1}{2} \alpha_{p, i}^{(2)} \alpha_{-p, i}^{(2)}
$$

Using the explicit form of the $2-2$ Neumann function $\tilde{N}_{m n}^{22}=\frac{(-1)^{m+n}}{4 \pi \mu\left|\alpha_{(1)}\right| y}$, we find that the prefactor is simply $2 \mu \alpha_{1}^{c l}=2 \mu$ and hence the matrix element of the interaction vertex is given as

$$
4 \mu \frac{\sqrt{J_{1} J_{2} J_{3}}}{N}\left(-\tilde{N}_{p,-p}^{22}\right)=-4 \mu \frac{\sqrt{J_{1} J_{2} J_{3}}}{N} \frac{1}{4 \pi \mu\left|\alpha_{(1)}\right| y} .
$$




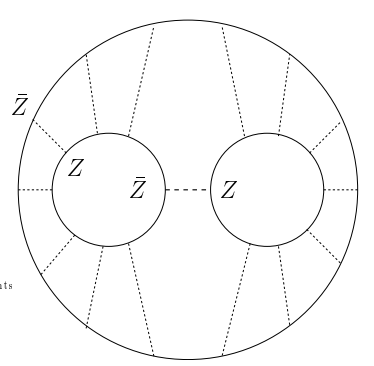

Figure 2: The contraction of $Z$ and $\bar{Z}$ between 2 and 3, with 2 being the singlet operator and 3 the ground state operator.

By multiplying the factor $G$

$$
\frac{1}{\mu\left(\Delta_{2}+\Delta_{3}-\Delta_{1}\right)}\left(f \frac{J_{2} J_{3}}{J_{1}}\right)^{-1} \Gamma\left(\frac{\Delta_{2}+\Delta_{3}-\Delta_{1}}{2}+1\right) \sim \frac{1}{2 \mu} \frac{4 \pi \mu\left|\alpha_{(1)}\right|}{J_{1}},
$$

we find that the holographic relation is precisely satisfied including numerical coefficient and sign.

This simplest example shows that the mixing of the second term (5.3) is absolutely necessary for this agreement, and also that it is responsible to the (2-2) Neumann functions on the string side. To convince the universality of this role of the $Z \bar{Z}$ mixing term, it is useful to treat the case where both of the operators 2 and 3 are singlet states. So, now $O^{(3)}$ in (5.2) is replaced by

$$
O_{s(q ;-q)}^{(3)}=\frac{1}{2 \sqrt{J_{3} N^{J_{3}+2}}} \operatorname{Tr}\left[\sum_{a=0}^{J_{3}} \phi_{i} Z^{a} \phi_{i} Z^{J_{3}-a} \mathrm{e}^{-\frac{2 \pi i}{J_{3}} a q}-4 \bar{Z} Z^{J_{3}+1}\right] .
$$

Then, the CFT coefficient consists of two contributions with different sets of free-field contractions as in fig. 3,

$$
C_{123}=C_{123}^{i i \leftrightarrow j j}+C_{123}^{Z \bar{Z}}
$$

corresponding to the contributions from the contractions between scalar excitations and between $Z, \bar{Z}$ fields, respectively. By similar calculations as above, we find

$$
C_{123}^{i i \leftrightarrow j j}=\frac{\sqrt{J_{1} J_{2} J_{3}}}{N} \frac{4}{J_{2} J_{3}}, \quad C_{123}^{Z \bar{Z}}=\frac{\sqrt{J_{1} J_{2} J_{3}}}{N} \frac{8}{J_{2} J_{3}} .
$$

On the string-field side, we find the following matrix element of the interaction vertex, by using self-explanatory notations,

$$
\lambda_{123}=\lambda_{123}^{2 \leftrightarrow 3}+\lambda_{123}^{\text {self }}
$$



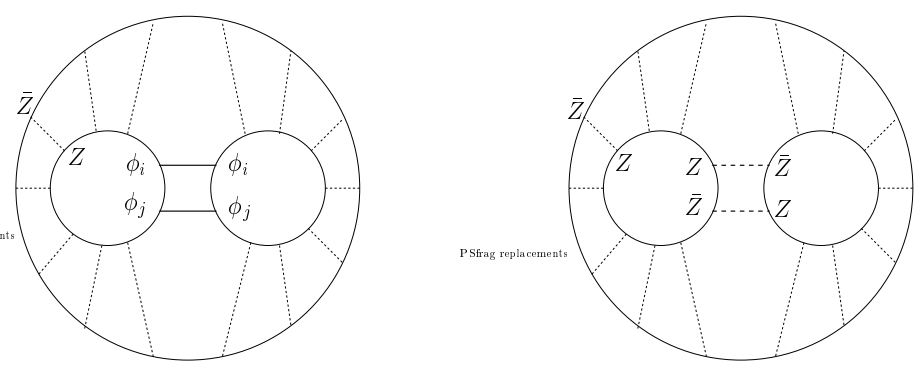

Figure 3: The two types of contractions between 2 and 3 which are both singlet operators with two scalar impurities.

with

$$
\begin{aligned}
& \lambda_{123}^{2 \leftrightarrow 3}=4 \mu \frac{\sqrt{J_{1} J_{2} J_{3}}}{N} \times 2\left(\tilde{N}_{p q}^{23}\right)^{2}=4 \mu \frac{\sqrt{J_{1} J_{2} J_{3}}}{N} \times 2\left(\frac{J_{1}}{4 \pi \mu\left|\alpha_{(1)}\right|}\right)^{2} \frac{1}{J_{2} J_{3}} \\
& \lambda_{123}^{\text {self }}=4 \mu \frac{\sqrt{J_{1} J_{2} J_{3}}}{N} \times 4 \tilde{N}_{p,-p}^{22} \tilde{N}_{q,-q}^{33}=4 \mu \frac{\sqrt{J_{1} J_{2} J_{3}}}{N} \times 4\left(\frac{J_{1}}{4 \pi \mu\left|\alpha_{(1)}\right|}\right)^{2} \frac{1}{J_{2} J_{3}}
\end{aligned}
$$

where we have again used the property that the Neumann functions appearing here are independent of the signs of momentum. Since the factor $G$ is

$$
\frac{1}{\mu\left(\Delta_{2}+\Delta_{3}-\Delta_{1}\right)}\left(f \frac{J_{2} J_{3}}{J_{1}}\right)^{-2} \Gamma\left(\frac{\Delta_{2}+\Delta_{3}-\Delta_{1}}{2}+1\right) \sim 2 \frac{1}{4 \mu}\left(\frac{4 \pi \mu\left|\alpha_{(1)}\right|}{J_{1}}\right)^{2},
$$

the results on both sides exactly match separately for two contributions between (5.8) and (5.10). These two exercises seem sufficient to convince the general validity of our holographic relation for class I processes involving singlet representation.

\subsection{Class II with scalar singlets}

Let us next consider an example of class II processes which are possible with the presence of trace part in the operator 1 . Consider the simplest nontrivial case with

$$
\begin{gathered}
O_{s(p ;-p)}^{(1)}=\frac{1}{2 \sqrt{J_{1} N^{J_{1}+2}}} \operatorname{Tr}\left[\sum_{a=0}^{J_{1}} \phi_{i} Z^{a} \phi_{i} Z^{J_{1}-a} \mathrm{e}^{-\frac{2 \pi i}{J_{1}} a p}-4 \bar{Z} Z^{J_{1}+1}\right] \\
O^{(2)}=\frac{1}{\sqrt{J_{2} N^{J_{2}}}} \operatorname{Tr}\left[Z^{J_{2}}\right], \quad O^{(3)}=\frac{1}{\sqrt{J_{3} N^{J_{3}}}} \operatorname{Tr}\left[Z^{J_{3}}\right] .
\end{gathered}
$$

On the gauge-theory side, there is no possibility of free contraction for either term of the operator 1. This means that the CFT coefficient vanish in the leading order at least in the $1 / \mu$-expansion and hence is at most of order $g_{2} \lambda^{\prime} \propto 1 / \mu^{2}$, if it does not vanish. 
On the other hand, the corresponding matrix element of the 3-point interaction vertex is given as, suppressing an obvious $\mathrm{SO}(4)$ indices,

$$
\begin{gathered}
\frac{\sqrt{J_{1} J_{2} J_{3}}}{N} \frac{\mu}{2}\langle 0| \alpha_{p}^{(1)} \alpha_{-p}^{(1)}\left(2+\alpha_{p}^{(1) \dagger} \alpha_{-p}^{(1)}+\alpha_{-p}^{(1) \dagger} \alpha_{p}^{(1)}\right) \\
\times \exp \left[-\frac{1}{2}\left(\tilde{N}_{p p}^{11}\left(\alpha_{p}^{(1) \dagger}\right)^{2}+\tilde{N}_{-p,-p}^{11}\left(\alpha_{-p}^{(1) \dagger}\right)^{2}\right)-\tilde{N}_{p,-p}^{11} \alpha_{p}^{(1) \dagger} \alpha_{-p}^{(1) \dagger}\right]|0\rangle \\
=-\frac{\sqrt{J_{1} J_{2} J_{3}}}{N} \frac{\mu}{2}\left(2 \tilde{N}_{p,-p}^{11}+\tilde{N}_{-p,-p}^{11}+\tilde{N}_{p p}^{11}\right) .
\end{gathered}
$$

The explicit form of the (11)-Neumann function is

$$
\tilde{N}_{n m}^{11}=\tilde{N}_{-n,-m}^{11}=-\tilde{N}_{-n, m}^{11}=-\tilde{N}_{n,-m}^{11}=(-1)^{m+n+1} \frac{\sin (\pi m y) \sin (\pi n y)}{\pi \mu\left|\alpha_{(1)}\right|}
$$

in the leading order in the $1 / \mu$ expansion. The correction terms for this expression is at most of order $1 /\left(\mu\left|\alpha_{(1)}\right|\right)^{3}$. Therefore, the matrix element vanishes in the leading order and possible corrections must be at most of order $\frac{\mu}{\left(\mu\left|\alpha_{(1)}\right|\right)^{3}}$.

Let us next examine the multiplying factor,

$$
G \equiv \frac{1}{\mu\left(\Delta_{2}+\Delta_{3}-\Delta_{1}\right)}\left(f \frac{J_{2} J_{3}}{J_{1}}\right)^{-\frac{\Delta_{2}+\Delta_{3}-\Delta_{1}}{2}} \Gamma\left(\frac{\Delta_{2}+\Delta_{3}-\Delta_{1}}{2}+1\right) .
$$

One of new features of class II processes is that $\alpha_{1}^{c l}=\frac{\Delta_{2}^{c l}+\Delta_{3}^{c l}-\Delta_{1}^{c l}}{2}$ is a negative integer. In the present example,

$$
\alpha_{1}^{c l}=-1
$$

which leads to the following leading behavior

$$
G \sim \frac{1}{\mu} f \frac{J_{2} J_{3}}{J_{1}} \frac{\left(\mu \alpha_{(1)}\right)^{2}}{p^{2}}=\frac{1}{\mu} \frac{J_{1}}{4 \pi \mu\left|\alpha_{(1)}\right|} \frac{\left(\mu \alpha_{(1)}\right)^{2}}{p^{2}}=\frac{J_{1}\left|\alpha_{(1)}\right|}{4 \pi p^{2}} .
$$

Note that we have here taken into account the singularity of the $\Gamma$-function $\Gamma(\epsilon) \sim 1 / \epsilon$. Thus we conclude that the CFT coefficient must be at most of order $1 /\left(\mu \alpha_{(1)}\right)^{2} \sim \lambda^{\prime}$ in conformity with the result from the gauge-theory side. We have again seen the crucial role played by the factor $G$ in relating both sides. The precise calculation of the higher order terms for 3-point correlation functions would require in general to take into account various mixing terms involving both bosonic and fermionic impurities. 


\subsection{Vector singlets}

The case of vector-singlet states is more subtle. The general prescription adopted in section 4 for dealing with traceless vector excitations is not applicable to this case, since in the presence of trace part there are a variety of different ways of constructing $\mathrm{SO}(4)$ invariants after taking spacetime derivatives comparing the case for purely non-singlet processes. We consider only some simple examples.

The simplest singlet operator with vector impurities with nonzero momenta $p$ and $-p$ is

$$
O_{v s(p ;-p)}=\frac{1}{4 \sqrt{J N^{J}}} \sum_{a=0}^{J-2} \operatorname{Tr}\left[\left(D_{j} Z\right) Z^{a}\left(D_{j} Z\right) Z^{J-2-a} \mathrm{e}^{-\frac{2 \pi i}{J-1} a p}\right]
$$

where the vector $\mathrm{SO}(4)$ index $j$ is summed over from 1 to 4 . The derivatives should now be computed in the standard way. It is easy to check that this satisfies the orthonormality. Note that we can ignore the second derivatives $D^{2} Z$ in the present approximation due to the equation of motion.

Let us consider the simplest example of class I, in which the operator 2 is this singlet state, while 1 and 3 are in the ground state,

$$
O_{v s(p ;-p)}^{(2)}=\frac{1}{4 \sqrt{J_{2} N_{2}^{J}}} \sum_{a=0}^{J_{2}-2} \operatorname{Tr}\left[\left(D_{j} Z\right) Z^{a}\left(D_{j} Z\right) Z^{J_{2}-2-a} \mathrm{e}^{-\frac{2 \pi i}{J_{2}-1} a p}\right] .
$$

Then, the free-field contractions between 1 and 2 yields the sum

$$
\sum_{a=0}^{J_{2}-2} \mathrm{e}^{-\frac{2 \pi i}{J_{2}-1} a p}=0 . \quad(p \neq 0)
$$

We conclude that the corresponding CFT coefficient must be zero in the leading order. On the side of string-field theory, the matrix element of the 3-point vertex is

$$
\begin{aligned}
-\frac{\sqrt{J_{1} J_{2} J_{3}}}{N} & \frac{\mu}{2}\langle 0| \alpha_{p}^{(2)} \alpha_{-p}^{(2)}\left(2-\alpha_{p}^{(2) \dagger} \alpha_{-p}^{(2)}-\alpha_{-p}^{(2) \dagger} \alpha_{p}^{(2)}\right) \\
& \times \exp \left[-\frac{1}{2} \tilde{N}_{p p}^{22}\left(\alpha_{p}^{(2) \dagger}\right)^{2}-\frac{1}{2} \tilde{N}_{-p,-p}^{22}\left(\alpha_{-p}^{(2) \dagger}\right)^{2}-\tilde{N}_{p,-p}^{22} \alpha_{p}^{(2) \dagger} \alpha_{-p}^{(2) \dagger}\right]|0\rangle
\end{aligned}
$$

which is equal to

$$
-\frac{\sqrt{J_{1} J_{2} J_{3}}}{N} 2 \mu\left(-2 \tilde{N}_{p,-p}^{22}+\tilde{N}_{p p}^{22}+\tilde{N}_{-p,-p}^{22}\right)=0
$$

by using explicit forms of the Neumann functions which has already been used for the case of scalar singlet. Note that the crucial relative sign in the prefactor in the first line in the expression (5.20), which is opposite to the corresponding scalar case. Since there is 

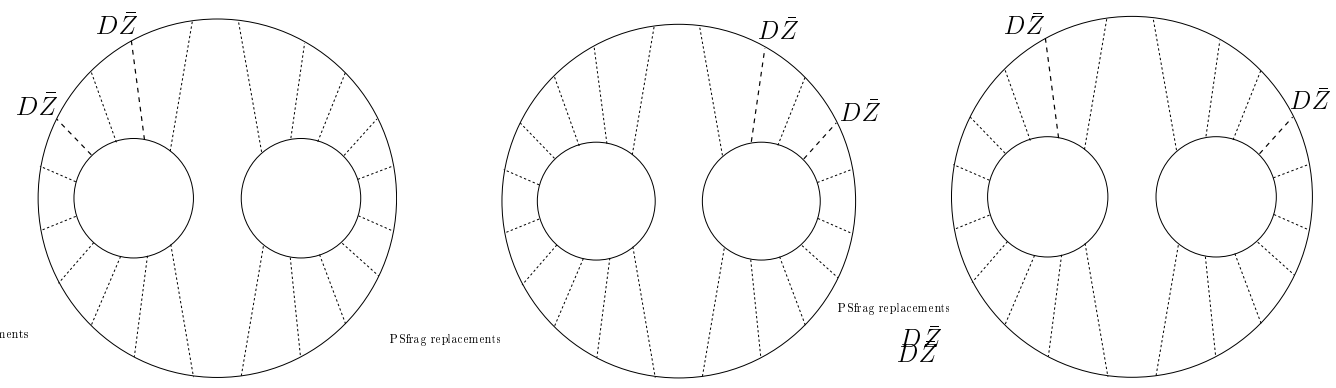

Figure 4: The three types of contractions for the class II process with singlet vector operator for the line 1.

no singularity in the factor $G$ in the holographic relation, this shows that the both sides match. It is easy to check that the vanishing of the CFT coefficient continues to be valid when we increase the number of singlet vector impurities in the lines 2 and 3 on both sides.

The situation drastically changes for the class II case. Consider the simplest such process where the operator 1 is the vector singlet state with 2 and 3 being in the ground state:

$$
O_{v s(p ;-p)}^{(1)}=\frac{1}{4 \sqrt{J_{1} N^{J_{1}}}} \sum_{a=0}^{J_{1}-2} \operatorname{Tr}\left[\left(D_{j} Z\right) Z^{a}\left(D_{j} \bar{Z}\right) Z^{J_{1}-2-a} \mathrm{e}^{-\frac{2 \pi i}{J_{1}-1} a p}\right] .
$$

On the gauge-theory side, the 3-point function consists of three different types of contributions

$$
\left\langle\bar{O}_{v s(p ;-p)}^{(1)}\left(\vec{x}_{1}\right) O^{(2)}\left(\vec{x}_{2}\right) O^{(3)}\left(\vec{x}_{3}\right)\right\rangle=F_{123}^{1 \leftrightarrow 2}+F_{123}^{1 \leftrightarrow 3}+F_{123}^{1 \leftrightarrow(2,3)}
$$

In the first and second terms, both of two derivatives in (5.22) acts only on $(1 \leftrightarrow 2)$ contractions and on $(1 \leftrightarrow 3)$ contractions, respectively. In the third contribution, one of the derivatives acts on $(1 \leftrightarrow 2)$ contractions and the other on $(1 \leftrightarrow 3)$ contractions. See fig. 4. We find

$$
\begin{aligned}
F_{123}^{1 \leftrightarrow 2}= & \frac{2}{N \sqrt{J_{1} J_{2} J_{3}}} J_{2} J_{3} \times J_{1}^{2} \frac{\sin ^{2} \pi y p}{(\pi p)^{2}} \frac{1}{\left|\vec{x}_{12}\right|^{2 J_{2}+2}\left|\vec{x}_{13}\right|^{2 J_{3}}}, \\
F_{123}^{1 \leftrightarrow 3}= & \frac{2}{N \sqrt{J_{1} J_{2} J_{3}}} J_{2} J_{3} \times J_{1}^{2} \frac{\sin ^{2} \pi(1-y) p}{(\pi p)^{2}} \frac{1}{\left|\vec{x}_{12}\right|^{2 J_{2}}\left|\vec{x}_{13}\right|^{2 J_{3}+2}}, \\
F_{123}^{1 \leftrightarrow(2,3)}= & \frac{4}{N \sqrt{J_{1} J_{2} J_{3}}} J_{2} J_{3} \\
& \quad \times J_{1}^{2}(-1)^{p} \frac{\sin \pi y p \sin \pi(1-y) p}{(\pi p)^{2}} \frac{\left(\vec{x}_{12} \cdot \vec{x}_{13}\right)}{\left|\vec{x}_{12}\right|^{2 J_{2}+2}\left|\vec{x}_{13}\right|^{2 J_{3}+2}} .
\end{aligned}
$$


Summing up these three contributions, we have the expression

$$
2 \frac{\sqrt{J_{1} J_{2} J_{3}}}{N} J_{1} \frac{\sin ^{2} \pi y p}{(\pi p)^{2}} \frac{\left|\vec{x}_{23}\right|^{2}}{\left|\vec{x}_{12}\right|^{2 J_{2}+2}\left|\vec{x}_{13}\right|^{2 J_{3}+2}} .
$$

The spacetime factor is precisely the form we should expect for a type II process with $\alpha_{1}^{c l}=-1$, and hence the CFT coefficient is

$$
C_{123}=2 \frac{\sqrt{J_{1} J_{2} J_{3}}}{N} J_{1} \frac{\sin ^{2} \pi y p}{(\pi p)^{2}}
$$

Let us turn to the string side. The matrix element of the interaction vertex is given by

$$
\begin{gathered}
-\frac{\sqrt{J_{1} J_{2} J_{3}}}{N} \frac{\mu}{2}\langle 0| \alpha_{p}^{(1)} \alpha_{-p}^{(1)}\left(2-\alpha_{p}^{(1) \dagger} \alpha_{-p}^{(1)}-\alpha_{-p}^{(1) \dagger} \alpha_{p}^{(1)}\right) \\
\times \exp \left[-\frac{1}{2}\left(\tilde{N}_{p p}^{11}\left(\alpha_{p}^{(1) \dagger}\right)^{2}+\tilde{N}_{-p,-p}^{11}\left(\alpha_{-p}^{(1) \dagger}\right)^{2}\right)-\tilde{N}_{p,-p}^{11} \alpha_{p}^{(1) \dagger} \alpha_{-p}^{(1) \dagger}\right]|0\rangle \\
=-4 \frac{\sqrt{J_{1} J_{2} J_{3}}}{N} \frac{\mu}{2}\left(-2 \tilde{N}_{p,-p}^{11}+\tilde{N}_{-p,-p}^{11}+\tilde{N}_{p p}^{11}\right)=\frac{\sqrt{J_{1} J_{2} J_{3}}}{N} \frac{8}{\pi\left|\alpha_{(1)}\right|} \sin ^{2}(\pi \mu p) .
\end{gathered}
$$

The difference from the scalar type II case treated in the previous subsection is of course the relative sign in the prefactor apart from the trivial difference of the suppressed internal indices. By multiplying the factor $G$ which is the same as (5.17), we confirm that the CFT coefficient (5.27) is precisely reproduced.

In the present paper, we have restricted our discussions only to bosonic excitations. If we consider fermionic excitations, the correlation functions generically start from higher orders in $1 / \mu$, since impurity non-preserving exchanges of BMN fermion fields with positive $\mathrm{U}(1)$-charge $1 / 2$ between 2 and 3 in general require Yukawa-type interactions with scalar fields because of charge conservation. Furthermore, we have to take into account complex mixings among operators which have different numbers of bosonic and fermionic excitations but with degenerate conformal dimensions. For a precise treatment of these phenomena, it is very important to formulate the correspondence of supersymmetries between bulk and boundary. Supersymmetry would put various constraints on the correlation functions among both bosonic and fermionic impurities. For examples of such constraints in the context of the pp-wave limit, see e.g. [14] [15] and references therein. We are planning to discuss these aspects separately in the next work. 


\section{Remarks}

In this final section, we give a few remarks on some relevant issues related to the main problem of the present work. We hope that this is useful for having further perspective on our approach.

\subsection{Uniqueness of the holographic relation and the integrability of string field theory}

The holographic relation summarized in section 2 was derived on the basis of the GKPWitten relation and its perturbative computation in the case of 3-point function using bulk-to-boundary propagator. The original effective action for supergravity modes on which our derivation is based is written in terms of a particular choice of fields [16 by which derivatives with respect to the $\mathrm{AdS}_{5}$ spacetime coordinates are completely eliminated. This is justified since the 3-point function is independent of field redefinition. To the present order of approximation, it is sufficient to show that a would-be interaction term which could be generated from the quadratic term by a field redefinition does not contribute to the 3-point function. For notational simplicity, let us take the case of a single scalar field $\phi$ with action

$$
\int d^{5} x \sqrt{g} \frac{1}{2}\left[(\partial \phi)^{2}+m^{2} \phi^{2}\right] .
$$

By a field redefinition $\phi \rightarrow \phi+c \phi^{2}$, a 3-point interaction vertex

$$
v_{3}=\sqrt{g} c \phi^{2}(\mathcal{K} \phi), \quad \mathcal{K}=-\frac{1}{\sqrt{g}} \partial_{i} \sqrt{g} \partial_{i}+m^{2}
$$

is generated. However, since the bulk-boundary propagator $\Delta(z, \vec{x} ; \vec{y})$ satisfies the equation of motion $\mathcal{K} \Delta(z, \vec{x} ; \vec{y})=0$ for arbitrary bulk spacetime positions $(z, \vec{x})$, we conclude that this vertex $v_{3}$ does not contribute to the correlation function, according to the GKPWitten prescription. Therefore, the holographic relation before the large- $J$ limit is unique.

Actually, in the special case of the so-called extremal correlators with $\Delta_{2}+\Delta_{3}-\Delta_{1}=0$, there is a well known subtlety. For generic configurations of $\mathrm{U}(1) R$-charge, this is possible only for protected supergravity modes. The on-shell matrix elements of 3-point interaction vertex in the bulk vanish for this case, but the 3-point correlation functions do not. In our formalism, the zero of the interaction vertex is canceled by the zero of the denominator in relating the interaction vertex to the CFT coefficient. In this sense, the extremal correlator has the $0 / 0$ ambiguity. However, we can circumvent this problem in the GKP-Witten 
relation and in our holographic relation if we slightly shift the conformal dimensions $\Delta_{i} \rightarrow \Delta_{i}+\epsilon_{i}$ by analytic continuation using the generic matrix elements of the interaction vertex such that the degeneracy $\Delta_{2}+\Delta_{3}=\Delta_{1}$ is lifted and, take the limit $\epsilon_{i} \rightarrow 0$ finally after we compute the CFT coefficients.

By taking the large- $J$ limit, we arrived at the first order action described in section 2 which describes the dynamics along a single tunneling trajectory. A question now arises as to the possibility of similar field redefinitions after the large- $J$ limit. Here we have to be very careful: In computing 3-point correlation functions using the tunneling trajectory, we had to introduce a cutoff near the boundary, since the approximation of a single trajectory is violated near the boundary even if we take a short distance limit by which two among three boundary points approach to each other up to a short distance of order $\delta$. Finding the precise prescription for this cutoff was the essence of section 3 of our previous work [2].** Namely, the integral with respect to the positions of interacting point along the tunneling trajectory in the naive perturbation theory

$$
\int_{-\infty}^{+\infty} d \tau \mathrm{e}^{-\left(\Delta_{1}-\Delta_{2}-\Delta_{3}\right) \tau}
$$

must be replaced by

$$
\int_{-\infty}^{+\infty} d \tau \mathrm{e}^{-\left(\Delta_{1}-\Delta_{2}-\Delta_{3}\right) \tau} \exp \left[-f \frac{J_{2} J_{3}}{J_{1}} \frac{(2 \delta)^{2}}{\left|\vec{x}_{1}-\vec{x}_{c}\right|^{2}} \mathrm{e}^{2 \tau}\right]
$$

where $f$ plays the role of taking into account the $\alpha^{\prime}$ corrections. This implies that we cannot ignore total derivatives of the type

$$
\frac{d}{d \tau} \mathrm{e}^{-\left(\Delta_{1}-\Delta_{2}-\Delta_{3}\right) \tau}=-\left(\Delta_{1}-\Delta_{2}-\Delta_{3}\right) \mathrm{e}^{-\left(\Delta_{1}-\Delta_{2}-\Delta_{3}\right) \tau}
$$

inside the integral. It should be remarked that for this integral to be well defined we have to assume an analytic continuation in $\Delta_{1}-\Delta_{2}-\Delta_{3}$. Thus the shift $\Delta_{i} \rightarrow \Delta_{i}+\epsilon_{i}$ of conformal dimensions which is necessary for the extremal case is actually a general premise for our approach. Furthermore, the short distance limit $\delta \rightarrow 0$ must be taken after evaluating the integral with $f \frac{J_{2} J_{3}}{J_{1}} \delta^{2}=f y(1-y) J_{1} \delta^{2}$ being kept fixed. In other

\footnotetext{
${ }^{* *}$ To avoid possible confusions, we emphasize that taking the short-distance limit $\delta \rightarrow 0$ here is different from considering double-trace operators. In our previous work 2, this limit was used for the purpose of extracting the 3-point OPE coefficients. Its connection with the mixing of double-trace operators for the impurity-preserving sector was clarified there. For impurity non-preserving processes, 3-point functions cannot be related to the mixing matrix of a dilatation operator, since by definition we are treating operators with definite conformal dimensions which are eigenstates of dilatation.
} 
words, the order of two limits, large $J$ and small $\delta$, must be carefully chosen such that the integral is meaningful. Note that by overall scaling $\vec{x}_{r} \rightarrow \vec{x}_{r} / \delta$ the short-distance limit can equivalently be regarded as a large-distance limit in which $\left|\vec{x}_{23}\right|$ is fixed while $\vec{x}_{1}$ is sent to infinity.

The expression (6.1) is basically why we cannot impose conservation of energy for our 'Euclidean' S-matrix. Recall that in the argument for the uniqueness of 3-point functions before the large- $J$ limit, total spacetime derivatives are assumed to be vanishing. That is justified in the case of the GKP-Witten relation since the integral with respect to the interaction point extends to the whole bulk spacetime. In contrast to this, in our tunneling picture emerging by taking the large- $J$ limit, we have to be very careful when total derivatives are involved.

In connection with this, it is appropriate to reconsider the suggestion made in our first work [3] concerning a possible integrable structure of holographic string field theory, namely, the interaction term is actually obtained from the free theory by making a similarity transformation expressed using the CFT coefficient. In the present first order approximation, this can equivalently be formulated by a canonical transformation

$$
\begin{aligned}
& \psi(\tau) \rightarrow \psi_{c}(\tau)=\psi(\tau)+\frac{1}{2} \tilde{C} \psi^{2}(\tau)-\tilde{C} \bar{\psi}(\tau) \psi(\tau) \\
& \bar{\psi}(\tau) \rightarrow \bar{\psi}_{c}(\tau)=\bar{\psi}(\tau)-\tilde{C} \psi(\tau) \bar{\psi}(\tau)+\frac{1}{2} \tilde{C} \bar{\psi}^{2}(\tau)
\end{aligned}
$$

where $\tilde{C}$ is proportional to the CFT coefficient itself

$$
\tilde{C}_{123}=\left(f \frac{J_{2} J_{3}}{J_{1}}\right)^{\left(\Delta_{2}+\Delta_{3}-\Delta_{1}\right) / 2} \frac{C_{123}}{\Gamma\left(\frac{\Delta_{2}+\Delta_{3}-\Delta_{1}}{2}+1\right)}
$$

because of the relation between the CFT coefficient and the 3-point coupling $\lambda_{123}$

$$
\left(H_{2}^{(2)}+H_{2}^{(3)}-H_{2}^{(1)}\right) \tilde{C}_{123}=\lambda_{123}
$$

Note that we are here using a symbolic notation for brevity. It should be a trivial task to convert those formal expressions using the bra-ket notation for string fields. As is easily seen, the canonical transformation, (6.2) and (6.3), generates the 3-point interaction term $\lambda_{123}$ from the free Hamiltonian. This shows that the equation of motion of our holographic string field theory is integrable provided that the CFT coefficient is well defined.

Does this imply that the three-point functions are obtained from the free string-field theory by the field redefinition corresponding to this canonical transformation? The 
answer to this question is clearly no, since the Euclidean S-matrix is invariant under field redefinition. Recall that the equivalence with respect to the equation of motion does not in general imply the equivalence of quantum mechanical amplitudes, because the surface terms often play crucial roles.

Let us briefly check this using the LSZ formalism. The 3-point S-matrix elements are defined as the residue of the 3-point Green functions $\left\langle\psi\left(\tau_{1}\right) \bar{\psi}\left(\tau_{2}\right) \bar{\psi}\left(\tau_{3}\right)\right\rangle$ at the poles at $\omega_{1}=-i \mu \Delta_{1}, \omega_{2}=i \mu \Delta_{2}$ and $\omega_{3}=i \mu \Delta_{3}$, respectively, in the complex-energy $\left(\omega_{i}, i=\right.$ $1,2,3)$ plane corresponding to external lines. If the field redefinition could generate a 3-point S-matrix element from the free theory, that would be obtained from the Green function

$$
\left\langle\psi_{c}\left(\tau_{1}\right) \bar{\psi}_{c}\left(\tau_{2}\right) \bar{\psi}_{c}\left(\tau_{3}\right)\right\rangle_{\text {free }}
$$

where we compute the Green function using the free field action for the original string fields $\psi(\tau)$ and $\bar{\psi}(\tau)$ (not for the transformed fields $\psi_{c}$ and $\bar{\psi}_{c}$ ). Assuming that the $R$ charge angular momenta of external states are $J_{1}\left(=J_{2}+J_{3}\right), J_{2}$ and $J_{3}$ respectively corresponding to our convention throughout the present work, it is sufficient to study the following part of this Green function,

$$
\left\langle\psi_{c}\left(\tau_{1}\right) \bar{\psi}\left(\tau_{2}\right) \bar{\psi}\left(\tau_{3}\right)\right\rangle_{\text {free }} \sim \frac{1}{2}\left\langle\tilde{C} \psi^{2}\left(\tau_{1}\right) \bar{\psi}\left(\tau_{2}\right) \bar{\psi}\left(\tau_{3}\right)\right\rangle_{\text {free }}=\tilde{C}\left\langle\psi\left(\tau_{1}\right) \bar{\psi}\left(\tau_{2}\right)\right\rangle_{\text {free }}\left\langle\psi\left(\tau_{1}\right) \bar{\psi}\left(\tau_{3}\right)\right\rangle_{\text {free }}
$$

Note that in this computation we do not use any partial integration with respect to the positions of interaction, since we do not make the change of field variables explicitly for the action itself. This expression has poles at the correct positions in the complex energy plane for the lines 2 and 3, but not for the line $1^{\dagger \dagger}$ except for the extremal case where the interaction vertex itself vanishes. As above, we assume the prescription $\Delta_{i} \rightarrow \Delta_{i}+\epsilon_{i}$ for treating the extremal correlators to remove the degeneracy. Under this definition, the Green functions in general, including the extremal case, do not have correct poles corresponding to external line 1, and hence the S-matrix elements vanish. Therefore, the free action cannot in general generate the S-matrix elements.

When we represent the Green function in the path-integral formalism, we would be able to discuss the above problem by making the change of the integrated string-fields from $(\psi, \bar{\psi})$ to the redefined ones $\left(\psi_{c}, \bar{\psi}_{c}\right)$. Then, it would transform the free action to

\footnotetext{
${ }^{\dagger \dagger}$ The cutoff (6.1) does not affect the positions of the leg poles.
} 


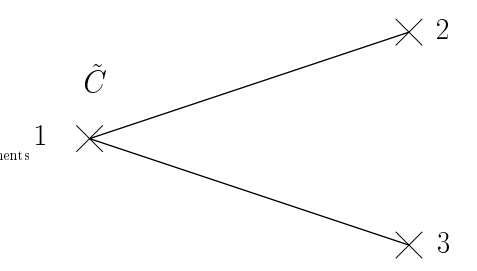

Figure 5: The diagram which contributes to the Green function of free string field after the field redefinition.

one with the interaction term and with crucial surface terms. The above direct treatment without using the change of the integration variables clearly shows that such an action generated by the canonical transformation does give only vanishing S-matrix, due to cancellation between local interaction terms and surface terms which are simultaneously generated by the change of integration variables. To summarize, the conclusion of this subsection is that our holographic relation is essentially unique, independently of possible field redefinitions, from both viewpoints of the large- $J$ limit of the GKP-Witten relation and of the logic of string field theory to the present order of approximations.

In passing, we note that the above ambiguity of the extremal case in the bulk S-matrix is essentially the same thing on the gauge-theory side as the ambiguity mentioned in [17] with respect to the mixing of sugra BMN operators with double-trace operators. As we have argued in 2], however, we should not mix the double-trace operators for sugra modes. But if one wishes, one could mix the double-trace operators and correspondingly make a field-redefinition appropriately such that one obtains the same 3-point extremal correlators on both sides. The degree of arbitrariness is the same on both sides. So the existence of this ambiguity itself may be regarded as the consistency of our Euclidean S-matrix interpretation of holographic mapping in the PP-wave limit. We do not however adopt this viewpoint since without analytic continuation the extremal correlators cannot be computed from string-field theory umambigously. This peculiar ambiguity is related to a well known accidental degeneracy among multi-particle states of massless particles with strictly collinear momenta. In any case, there is no ambiguity at all for impurity non-preserving cases treated in the present paper.

\subsection{Higher-order effects}

Extension of our ideas to higher orders is an important next problem. In the present paper, we have only treated the leading order results with respect to $1 / \mu$-expansion (more 
precisely, $1 / \mu \alpha_{(r)} \sim R^{2} / J$-expansion) to the first order in the genus-expansion parameter $1 / N$. But in principle our holographic relation should be valid to all order in $1 / \mu$ within the planar approximation. Actually, it is straightforward to compute next or next-tonext orders on the string side, since the Neumann functions are known [18] 20] apart from nonperturbative contributions. Corresponding computations on the gauge-theory side, however, require us to obtain 3-point functions at least up to 2-loop orders, including all mixing effects. Once we go to such higher-orders, an interesting puzzle arises. The factor $G$ which played crucial roles in the foregoing discussions gives corrections of the form $\mu^{-2 n} \log ^{m} \mu(n>0)$, since the large $\mu$ behavior (2.10) of the function $f$ is correct up to nonperturbative exponential corrections, according to the above references. If we ignore the would-be nonperturbative terms of the Neumann functions, this implies that, when they are not protected, 3-point correlation functions are subject to these peculiar corrections which cannot be derived by the ordinary perturbation theory on the gaugetheory side.

At present, we are not sure how this should be interpreted. A possibility may be that the total sum of nonperturbative corrections on the Neumann functions might generate such logarithmic terms and cancel these corrections when $m \neq 0$. For possible subtleties of nonperturbative corrections, we refer the reader to [19] and also to [20]. Or, if such terms with nonzero $m$ are not canceled on the string side, nonperturbative effects (or exact summation over the whole perturbation series) both on the gauge-theory side and on the string side might be responsible for complete understanding of string/gauge duality. Of course, it is also possible that our holographic relation itself may be subject to corrections.

The higher orders with respect to $1 / N$ is also important. Since our holographic relation is originally derived for sugra modes in the planar approximation, it is not immediately clear whether our conjecture should be valid for nonplanar cases. In principle, we have to examine the same limiting procedure for such cases as we have discussed for planar 3-point functions. Following our philosophy, all rules of the holographic mapping should be derived on the basis of the correspondence between correlation functions on both sides. As for higher-genus corrections, it is not evident whether the computation of loop corrections on the string side can be closed within the PP-wave in the presence of the sum over intermediate states. Recently, it has been reported [21] that the energy shift computed from the $\left|H_{3}\right\rangle_{S V}$-type string vertex disagrees with the gauge-theory prediction 
even at the leading order in the first non-planar correction, if the intermediate states are restricted to impurity-preserving sector.

Even if it could be closed within the PP-wave limit, we have to fix possible higher-order corrections for susy generators and Hamiltonians themselves on the string field side. In our first paper [3] in this series, we have suggested that in planar (tree in the sense of string field theory) approximation, the higher-point vertices may be derived by a similarity transformation from free theory whose lowest-order form is nothing but the canonical transformation discussed in the previous subsection. If we include non-planar corrections, these (similarity or perhaps unitary in the space of string fields) transformations generate $1 / N$ corrections for vertices and even for quadratic kinetic terms. In order to confirm this suggestion, it is important to investigate first the extension of our arguments for higher-point amplitudes in the planar limit. It has been pointed out that the structure of 4-point correlation functions on the gauge-theory side suffers from some ambiguity [17] in determining the large- $J$ limit. From our point of view, we have to take into account the fact that the approximation of a single tunneling trajectory can be justified only if we first take the short-distance limit for operator products such that the order $\delta$ of the distances at each set of operator products at initial and final points at the boundary satisfies $\delta^{2} \sim c / J$ with some finite $c$ and finally let $c \rightarrow 0$. It is clear that the naive limit $J \rightarrow \infty$ on fixing the space-time configuration of operators at the boundary, which indeed leads to discontinuous results depending on the positions of operators, is not allowed. Careful analyses are required, however, to make more precise the general structure suggested in 3] for holographic mapping for higher-point S-matrix elements.

Note added: After completing this manuscritpt, we received a preliminary manuscript by H. Shimada 22] who tried to establish the holographic relation in an approach which is slightly different from ours. His argument and result, though restricted yet to impuritypreserving sector with scalar excitations, seem to be consistent with ours in [2] and complementary to our argument for the uniqueness of the holographic relation.

\section{Acknowledgements}

We would like to thank H. Shimada for discussions. The present work is supported in part by Grant-in-Aid for Scientific Research (No. 13135205 (Priority Areas) and No. 16340067 (B)) from the Ministry of Education, Science and Culture. 


\section{A Large $\mu$ behavior of Neumann coefficients}

We summarize here the leading large $\mu$ behavior of $\widetilde{N}_{m n}^{r s}$ for reader's convenience following [18, [20]:

$$
\begin{aligned}
& \widetilde{N}_{m n}^{22}=\frac{(-1)^{m+n}}{4 \pi \mu\left|\alpha_{(1)}\right| y}, \quad \widetilde{N}_{m n}^{23}=\frac{(-1)^{m+1}}{4 \pi \mu\left|\alpha_{(1)}\right| \sqrt{y(1-y)}} \\
& \widetilde{N}_{m n}^{33}=\frac{1}{4 \pi \mu\left|\alpha_{(1)}\right|(1-y)}, \quad \widetilde{N}_{m n}^{11}=\frac{(-1)^{m+n+1} \sin (\pi m y) \sin (\pi n y)}{\pi \mu\left|\alpha_{(1)}\right|} \\
& \widetilde{N}_{m n}^{21}=\frac{(-1)^{m+n+1} \sin (\pi n y)}{\pi \sqrt{y}(n-m / y)}, \quad \widetilde{N}_{m n}^{31}=\frac{(-1)^{n} \sin (\pi n y)}{\pi \sqrt{1-y}(n-m /(1-y))}
\end{aligned}
$$

for $(m, n) \neq(0,0)$, and

$$
\begin{aligned}
& \widetilde{N}_{00}^{11}=0, \quad \widetilde{N}_{00}^{12}=-\sqrt{y}, \quad \widetilde{N}_{00}^{13}=-\sqrt{1-y} \\
& \widetilde{N}_{00}^{23}=-\frac{1}{4 \pi \mu\left|\alpha_{(1)}\right| \sqrt{y(1-y)}}, \quad \widetilde{N}_{00}^{22}=\frac{1}{4 \pi \mu\left|\alpha_{(1)}\right| y}, \quad \widetilde{N}_{00}^{33}=\frac{1}{4 \pi \mu\left|\alpha_{(1)}\right|(1-y)},
\end{aligned}
$$

for $m=0=n$.

The asymptotic form in the large $\mu$ limit of $f=1-4 \mu \alpha_{(1)} \alpha_{(2)} \alpha_{(3)} K$ is given by

$$
f=\frac{1}{4 \pi \mu\left|\alpha_{(1)}\right| y(1-y)} .
$$

\section{References}

[1] D. Berenstein, J. M. Maldacena, H. Nastase, Strings in flat space and $p p$ waves from N=4 super Yang-Mills, JHEP0204(2002)013, hep-th/0202021.

[2] S. Dobashi and T. Yoneya, Resolving the holography in the plane-wave limit of AdS/CFT correspondence, hep-th/0406225.

[3] S. Dobashi, H. Shimada, and T. Yoneya, Holographic reformulation of string theory on $A d S_{5} \times S^{5}$ background in the PP-wave limit, Nucl.Phys.B665(2003)94, hep-th/0209251.

[4] T. Yoneya, What is holography in the plane wave limit of $A d S(5) / S Y M(4)$ correspondence?, hep-th/0304183 (expanded from the paper published in the Proceedings, Prog. Theor. Phys. 152 (2003) 108).

[5] M. Asano, Y. Sekino, and T. Yoneya, PP wave holography for Dp-brane backgrounds, Nucl.Phys.B678(2004)197, hep-th/0308024. M. Asano and Y. Sekino, Large $N$ limit of SYM theories with 16 supercharges from superstrings on Dp-brane backgrounds, hep-th/0405203 M. Asano, Stringy effect of the holographic correspondence for Dp-brane backgrounds hep-th/0408030. 
[6] M. Spradlin and A. Volovich, Superstring interactions in a pp wave background, Phys.Rev.D66(2002)086004,, hep-th/0204146 M. Spradlin and A. Volovich, Superstring interactions in a pp wave background 2, JHEP0301(2003)036, hep-th/0206073.

A. Pankiewicz, More comments on superstring interactions in the $p p$ wave background, JHEP0209(2002)056, hep-th/0208209 A. Pankiewicz and B. Stefanski,Jr., PP wave light cone superstring field theory, Nucl.Phys.B657(2003)79, hep-th/0210246 A. Pankiewicz, An alternative formulation of light cone string field theory on the plane wave, JHEP0306(2003)047, hep-th/0304232 A. Pankiewicz and B. Stefanski,Jr., On the uniqueness of plane wave string field theory, hep-th/0308062.

[7] P. Di Vecchia, J.L. Petersen, M. Petrini, R. Russo, and A. Tanzini, The three string vertex and the AdS/CFT duality in the pp wave limit, hep-th/0304025.

[8] C.S. Chu, V.V. Khoze, Correspondence between the three point BMN correlators and the three string vertex on the pp wave, JHEP0304(2003)014, hep-th/0301036.

[9] N. Beisert, C. Kristjansen, J. Plefka, G.W. Semenoff, and M. Staudacher, BMN correlators and operator mixing in N=4 super Yang-Mills theory, Nucl.Phys.B650(2003)125-161, hep-th/0208178

[10] N.R. Constable, D.Z. Freedman, M. Headrick, and S.Minwalla, Operator mixing and the BMN correspondence, JHEP0210(2002)068, hep-th/0209002

[11] N.R. Constable, D.Z. Freedman, M.Headrick, S. Minwalla, L. Motl, A. Postnikov, and W. Skiba, PP wave string interactions from perturbative Yang-Mills theory, JHEP0207(2002)017, hep-th/0205089.

[12] J. Gomis, S. Moriyama, and J. Park SYM description of SFT Hamiltonian in a pp wave background, Nucl.Phys.B659(2003)179, hep-th/0210153; J. Gomis, S. Moriyama, and J. Park SYM description of pp wave string interactions: singlet sector and arbitrary impurities, Nucl.Phys.B665(2003)49, hep-th/0301250

[13] A. Parnachev and A.V. Ryzhov, Strings in the near plane wave background and AdS/CFT, JHEP0210(2002)066, hep-th/0208010.

[14] N. Beisert, BMN operators and superconformal symmetry, Nucl.Phys.B659(2003)79, hep-th/0211032.

[15] U. Gürsoy, Vector operators in the BMN correspondence, JHEP0307:048,2003.

[16] S.-M. Lee, S. Minwalla, M. Rangamani, and N. Seiberg, Three point functions of chiral operators in $D=4, N=4 S Y M$ at large $N$, Adv.Theor.Math.Phys.2(1998)697, hep-th/9806074.

[17] C. Kristjansen, J. Plefka, G.W. Semenoff, and M. Staudacher, A new double scaling limit of $N=4$ super Yang-Mills theory and pp wave strings, Nucl.Phys.B643(2002)3, hep-th/0205033.

[18] Y. H, He, J. H. Schwarz, M. Spradlin, and A. Volovich, Explicit formulas for Neumann coefficients in the plane wave geometry, Phys.Rev.D67(2003)086005, hep-th/0211198.

[19] I. R. Klebanov, M. Spradlin, and A. Volovich, New Effects in Gauge Theory from pp-wave Superstrings, Phys. Lett. B548 (2002)111, hep-th/0206221. 
[20] J. Lucietti, S. Schafer-Nameki, and A. Sinha, On the plane-wave cubic vertex, Phys.Rev.D70(2004)026005, hep-th/0402185

[21] P. Gutjahr and A. Pankiewicz, New aspects of the BMN correspondence beyond the planar limit, hep-th/0407098.

[22] H. Shimada, to appear. 